\title{
Norm of a Bethe vector and the Hessian of the master function
}

\author{
Evgeny Mukhin and Alexander Varchenko
}

\begin{abstract}
We show that the norm of a Bethe vector in the $s l_{r+1}$ Gaudin model is equal to the Hessian of the corresponding master function at the corresponding critical point. In particular the Bethe vectors corresponding to non-degenerate critical points are non-zero vectors. This result is a byproduct of functorial properties of Bethe vectors studied in this paper. As another byproduct of functoriality we show that the Bethe vectors form a basis in the tensor product of several copies of first and last fundamental $s l_{r+1}$-modules.
\end{abstract}

\section{Introduction}

The Bethe ansatz is a large collection of methods in the theory of quantum integrable models to calculate the spectrum and eigenvectors for a certain commutative sub-algebra of observables for an integrable model. Elements of the sub-algebra are called hamiltonians, or integrals of motion, or conservation laws of the model. The bibliography on the Bethe ansatz method is enormous; see for example [BIK93, Fad90, FT79].

In the theory of the Bethe ansatz one assigns the Bethe ansatz equations to an integrable model. Then a solution of the Bethe ansatz equations gives an eigenvector of commuting hamiltonians of the model. The general conjecture is that the constructed vectors form a basis in the space of states of the model.

The simplest and most interesting example is the Gaudin model associated with a complex simple Lie algebra $\mathfrak{g}$; see [Bab93, BF94, Fre95, Fre04, FFR94, Gau76, MV00, RV95, SV03, Var95]. One considers highest weight $\mathfrak{g}$-modules $V_{\Lambda_{1}}, \ldots, V_{\Lambda_{n}}$ and their tensor product $V_{\boldsymbol{\Lambda}}$. One fixes a point $z=\left(z_{1}, \ldots, z_{n}\right) \in \mathbb{C}^{n}$ with distinct coordinates and defines linear operators $K_{1}(z), \ldots, K_{n}(z)$ on $V_{\boldsymbol{\Lambda}}$ by the formula

$$
K_{i}(z)=\sum_{j \neq i} \frac{\Omega^{(i, j)}}{z_{i}-z_{j}}, \quad i=1, \ldots, n .
$$

Here $\Omega^{(i, j)}$ is the Casimir operator acting in the $i$ th and $j$ th factors of the tensor product. The operators are called the Gaudin hamiltonians of the Gaudin model associated with $V_{\boldsymbol{\Lambda}}$. The hamiltonians commute.

The common eigenvectors of the Gaudin hamiltonians are constructed by the Bethe ansatz method. Namely, one assigns to the model a scalar function $\Phi(t, z)$ of new auxiliary variables $t$ and a $V_{\boldsymbol{\Lambda}}$-valued function $\omega(t, z)$ such that $\omega\left(t^{0}, z\right)$ is an eigenvector of the hamiltonians if $t^{0}$ is a critical point of $\Phi$. The functions $\Phi$ and $\omega$ were introduced in [SV91] to construct hypergeometric solutions

Received 8 March 2004, accepted in final form 7 August 2004, published online 21 June 2005.

2000 Mathematics Subject Classification 82B23 (primary), 17B67, 14M15 (secondary).

Keywords: Bethe ansatz, master functions, critical points.

The first author was supported in part by NSF grant DMS-0140460. The second author was supported in part by NSF grant DMS-0244579.

This journal is (C) Foundation Compositio Mathematica 2005. 


\section{Norm of A Bethe vector And the Hessian}

of the Knizhnik-Zamolodchikov (KZ) equations. The function $\Phi$ is called the master function and the function $\omega$ is called the universal weight function.

The first question is if the Bethe eigenvector $\omega\left(t^{0}, z\right)$ is non-zero. In this paper we show that for the $s l_{r+1}$ Gaudin model the Bethe vector is non-zero if $t^{0}$ is a non-degenerate critical point of the master function $\Phi$. To show that, we prove the following identity:

$$
S\left(\omega\left(t^{0}, z\right), \omega\left(t^{0}, z\right)\right)=\operatorname{Hess}_{t} \log \Phi\left(t^{0}, z\right) .
$$

Here $S$ is the tensor Shapovalov form on the tensor product $V_{\boldsymbol{\Lambda}}$ and the right-hand side of the formula is the Hessian at $t^{0}$ of the function $\log \Phi$. This formula for $s l_{2}$ Gaudin models was proved in [Var95]; see also [Kor82, Res86, RV95, TV96, MV00].

In this paper we prove the Bethe ansatz conjecture for tensor products of several copies of first and last fundamental $s l_{r+1}$-modules. Namely, we assume that $V_{\Lambda_{1}}, \ldots, V_{\Lambda_{n}}$ are $s l_{r+1}$-modules, each of which is either the first or last fundamental $s l_{r+1}$-module; then we show that for generic $z$ the Bethe vectors form an eigenbasis of the Gaudin hamiltonians in the tensor product $V_{\boldsymbol{\Lambda}}$. Note that $s l_{3}$ has only two fundamental modules: the first and last.

We also prove the Bethe ansatz conjecture for tensor products of several copies of arbitrary fundamental representations of $s l_{4}$.

The Bethe ansatz conjecture for $s l_{r+1}$ is related to the question of transversality of special Schubert cycles in the Grassmannian of $(r+1)$-dimensional planes in the space of polynomials of one variable; for more about this relation see $[\mathrm{MV} 04, \S 4]$ and for the corresponding transversality statements see [Sot99] and [EH83].

The formulated results are based on functorial properties of the master function and the universal weight function studied in this paper. Namely we study the behavior of $\Phi$ and $\omega$ when some of the coordinates of $z$ tend to the same limit. That corresponds to the situation in which the number of factors in the tensor product $V_{\boldsymbol{\Lambda}}$ becomes smaller while the factors become bigger. It turns out that under this limit the Bethe vectors behave in a reasonable way. That reasonable behavior allows us to establish some general properties of Bethe vectors under the condition that those properties hold for some model examples. The properties for the model examples can be checked by direct calculations. Ideas of that type were exploited earlier in [RV95].

The results of this paper split into two parts: one of them (constructions) is related to any simple Lie algebra $(\S \S 2-4)$; the other one $(\S \S 5-7)$ is related to $s l_{r+1}$ and can be considered as applications or examples of the previous constructions.

The paper is organized as follows. Section 2 contains the definitions of the master function and universal weight function. We prove there that the universal weight function is well defined on critical points of the master function. In $\S 3$ we collect information on iterated singular vectors in tensor products of representations. The functorial properties of the master and universal weight functions are studied in $\S 4$. Preliminary information on Bethe vectors and their Shapovalov norms is collected in $\S 5$. In $\S 6$ we prove Theorem 6.1 that the Bethe vectors form a basis in the tensor product of several copies of first and last fundamental $s l_{r+1}$-modules for generic $z$. In $\S 7$ we prove formula (1) using Theorem 6.1.

\section{Bethe vectors}

\subsection{The Gaudin model}

Let $\mathfrak{g}$ be a simple Lie algebra over $\mathbb{C}$ with Cartan matrix $A=\left(a_{i, j}\right)_{i, j=1}^{r}$. Let $D=\operatorname{diag}\left\{d_{1}, \ldots, d_{r}\right\}$ be the diagonal matrix with positive relatively prime integers $d_{i}$ such that $B=D A$ is symmetric. 


\section{E. Mukhin And A. VArchenko}

Let $\mathfrak{h} \subset \mathfrak{g}$ be the Cartan sub-algebra. Fix simple roots $\alpha_{1}, \ldots, \alpha_{r}$ in $\mathfrak{h}^{*}$ and an invariant bilinear form $($,$) on \mathfrak{g}$ such that $\left(\alpha_{i}, \alpha_{j}\right)=d_{i} a_{i, j}$. Let $H_{1}, \ldots, H_{r} \in \mathfrak{h}$ be the corresponding coroots, $\left\langle\lambda, H_{i}\right\rangle=$ $2\left(\lambda, \alpha_{i}\right) /\left(\alpha_{i}, \alpha_{i}\right)$ for $\lambda \in \mathfrak{h}^{*}$. In particular, $\left\langle\alpha_{j}, H_{i}\right\rangle=a_{i, j}$. Let $w_{1}, \ldots, w_{r} \in \mathfrak{h}^{*}$ be the fundamental weights, $\left\langle w_{i}, H_{j}\right\rangle=\delta_{i, j}$.

Let $E_{1}, \ldots, E_{r} \in \mathfrak{n}_{+}, H_{1}, \ldots, H_{r} \in \mathfrak{h}, F_{1}, \ldots, F_{r} \in \mathfrak{n}_{-}$be the Chevalley generators of $\mathfrak{g}$,

$$
\begin{aligned}
{\left[E_{i}, F_{j}\right] } & =\delta_{i, j} H_{i}, & & i, j=1, \ldots, r, \\
{\left[h, h^{\prime}\right] } & =0, & & h, h^{\prime} \in \mathfrak{h}, \\
{\left[h, E_{i}\right] } & =\left\langle\alpha_{i}, h\right\rangle E_{i}, & & h \in \mathfrak{h}, i=1, \ldots, r, \\
{\left[h, F_{i}\right] } & =-\left\langle\alpha_{i}, h\right\rangle F_{i}, & & h \in \mathfrak{h}, i=1, \ldots, r,
\end{aligned}
$$

and

$$
\left(\operatorname{ad} E_{i}\right)^{1-a_{i, j}} E_{j}=0, \quad\left(\operatorname{ad} F_{i}\right)^{1-a_{i, j}} F_{j}=0,
$$

for all $i \neq j$. have

Let $\left(x_{i}\right)_{i \in I}$ be an orthonormal basis in $\mathfrak{g}$, and $\Omega=\sum_{i \in I} x_{i} \otimes x_{i} \in \mathfrak{g} \otimes \mathfrak{g}$ the Casimir element. We

$$
[x \otimes 1+1 \otimes x, \Omega]=0
$$

in $U(\mathfrak{g}) \otimes U(\mathfrak{g})$ for any $x \in \mathfrak{g}$. Here $U(\mathfrak{g})$ is the universal enveloping algebra of $\mathfrak{g}$.

For a $\mathfrak{g}$-module $V$ and $\mu \in \mathfrak{h}^{*}$ denote by $V[\mu]$ the weight subspace of $V$ of weight $\mu$ and by Sing $V[\mu]$ the subspace of singular vectors of weight $\mu$,

$$
\text { Sing } V[\mu]=\left\{v \in V \mid \mathfrak{n}_{+} v=0, h v=\langle\mu, h\rangle v\right\} .
$$

Let $n$ be a positive integer and $\boldsymbol{\Lambda}=\left(\Lambda_{1}, \ldots, \Lambda_{n}\right), \Lambda_{i} \in \mathfrak{h}^{*}$, a set of weights. For $\mu \in \mathfrak{h}^{*}$ let $V_{\mu}$ be the irreducible $\mathfrak{g}$-module with highest weight $\mu$. Denote by $V_{\boldsymbol{\Lambda}}$ the tensor product $V_{\Lambda_{1}} \otimes \cdots \otimes V_{\Lambda_{n}}$.

If $X \in \operatorname{End}\left(V_{\Lambda_{i}}\right)$, then we denote by $X^{(i)} \in \operatorname{End}\left(V_{\boldsymbol{\Lambda}}\right)$ the operator $\cdots \otimes$ id $\otimes X \otimes \mathrm{id} \otimes \cdots$ acting non-trivially on the $i$ th factor of the tensor product. If $X=\sum_{k} X_{k} \otimes Y_{k} \in \operatorname{End}\left(V_{\Lambda_{i}} \otimes V_{\Lambda_{j}}\right)$, then we set $X^{(i, j)}=\sum_{k} X_{k}^{(i)} \otimes Y_{k}^{(j)} \in \operatorname{End}\left(V_{\boldsymbol{\Lambda}}\right)$.

Let $z=\left(z_{1}, \ldots, z_{n}\right)$ be a point in $\mathbb{C}^{n}$ with distinct coordinates. Introduce linear operators $K_{1}(z), \ldots, K_{n}(z)$ on $V_{\boldsymbol{\Lambda}}$ by the formula

$$
K_{i}(z)=\sum_{j \neq i} \frac{\Omega^{(i, j)}}{z_{i}-z_{j}}, \quad i=1, \ldots, n .
$$

The operators are called the Gaudin hamiltonians of the Gaudin model associated with $V_{\boldsymbol{\Lambda}}$. One can check directly that the hamiltonians commute, $\left[K_{i}(z), K_{j}(z)\right]=0$ for all $i, j$.

The main problem for the Gaudin model is to diagonalize simultaneously the hamiltonians; see [Bab93, BF94, Fre95, Fre04, FFR94, Gau76, MV00, RV95, SV03, Var95].

One can check that the hamiltonians commute with the action of $\mathfrak{g}$ on $V_{\boldsymbol{\Lambda}},\left[K_{i}(z), x\right]=0$ for all $i$ and $x \in \mathfrak{g}$. Therefore it is enough to diagonalize the hamiltonians on the subspaces of singular vectors $\operatorname{Sing} V_{\boldsymbol{\Lambda}}[\mu] \subset V_{\boldsymbol{\Lambda}}$.

The eigenvectors of the Gaudin hamiltonians are constructed by the Bethe ansatz method. We recall the construction in the next section.

\subsection{Master functions, critical points, and the universal weight function}

Fix a collection of weights $\boldsymbol{\Lambda}=\left(\Lambda_{1}, \ldots, \Lambda_{n}\right), \Lambda_{i} \in \mathfrak{h}^{*}$, and a collection of non-negative integers $\boldsymbol{l}=\left(l_{1}, \ldots, l_{r}\right)$. Denote $l=l_{1}+\cdots+l_{r}, \Lambda=\Lambda_{1}+\cdots+\Lambda_{n}$, and $\alpha(\boldsymbol{l})=l_{1} \alpha_{1}+\cdots+l_{r} \alpha_{r}$. 


\section{Norm of A Bethe vector AND the Hessian}

Let $c$ be the unique non-decreasing function from $\{1, \ldots, l\}$ to $\{1, \ldots, r\}$ such that $\# c^{-1}(i)=l_{i}$ for $i=1, \ldots, r$. The master function $\Phi(t, z, \boldsymbol{\Lambda}, \boldsymbol{l})$ is defined by

$$
\Phi(t, z, \boldsymbol{\Lambda}, \boldsymbol{l})=\prod_{1 \leqslant i<j \leqslant n}\left(z_{i}-z_{j}\right)^{\left(\Lambda_{i}, \Lambda_{j}\right)} \prod_{i=1}^{l} \prod_{s=1}^{n}\left(t_{i}-z_{s}\right)^{-\left(\alpha_{c(i)}, \Lambda_{s}\right)} \prod_{1 \leqslant i<j \leqslant l}\left(t_{i}-t_{j}\right)^{\left(\alpha_{c(i)}, \alpha_{c(j)}\right)}
$$

(see [SV91]). The function $\Phi$ is a function of complex variables $t=\left(t_{1}, \ldots, t_{l}\right), z=\left(z_{1}, \ldots, z_{n}\right)$, weights $\boldsymbol{\Lambda}$, and discrete parameters $\boldsymbol{l}$. The main variables are $t$; the other variables will be considered as parameters.

For given $z, \boldsymbol{\Lambda}, \boldsymbol{l}$, a point $t$ with complex coordinates is called a critical point of the master function if the following system of algebraic equations is satisfied

$$
-\sum_{s=1}^{n} \frac{\left(\alpha_{c(i)}, \Lambda_{s}\right)}{t_{i}-z_{s}}+\sum_{j, j \neq i} \frac{\left(\alpha_{c(i)}, \alpha_{c(j)}\right)}{t_{i}-t_{j}}=0, \quad i=1, \ldots, l .
$$

In other words, $t$ is a critical point if

$$
\left(\Phi^{-1} \frac{\partial \Phi}{\partial t_{i}}\right)(t)=0, \quad \text { for } i=1, \ldots, l .
$$

By definition, if $t=\left(t_{1}, \ldots, t_{l}\right)$ is a critical point and $\left(\alpha_{c(i)}, \alpha_{c(j)}\right) \neq 0$ for some $i, j$, then $t_{i} \neq t_{j}$. Also if $\left(\alpha_{c(i)}, \Lambda_{s}\right) \neq 0$ for some $i, s$, then $t_{i} \neq z_{s}$.

Let $\Sigma_{l}$ be the permutation group of the set $\{1, \ldots, l\}$. Denote by $\boldsymbol{\Sigma}_{\boldsymbol{l}} \subset \Sigma_{l}$ the subgroup of all permutations preserving the level sets of the function $c$. The subgroup $\boldsymbol{\Sigma}_{\boldsymbol{l}}$ is isomorphic to $\Sigma_{l_{1}} \times \cdots \times \Sigma_{l_{r}}$ and acts on $\mathbb{C}^{l}$ permuting coordinates of $t$. The action of the subgroup $\boldsymbol{\Sigma}_{\boldsymbol{l}}$ preserves the critical set of the master function. All orbits of $\boldsymbol{\Sigma}_{l}$ on the critical set have the same cardinality $l_{1} ! \cdots l_{r} !$.

Consider highest weight irreducible $\mathfrak{g}$-modules $V_{\Lambda_{1}}, \ldots, V_{\Lambda_{n}}$, the tensor product $V_{\boldsymbol{\Lambda}}=V_{\Lambda_{1}} \otimes \cdots \otimes$ $V_{\Lambda_{n}}$, and its weight subspace $V_{\boldsymbol{\Lambda}}[\Lambda-\alpha(\boldsymbol{l})]$. Fix a highest weight vector $v_{\Lambda_{i}}$ in $V_{\Lambda_{i}}$ for all $i$.

We construct a rational map

$$
\omega: \mathbb{C}^{l} \times \mathbb{C}^{n} \rightarrow V_{\boldsymbol{\Lambda}}[\Lambda-\alpha(\boldsymbol{l})]
$$

called the universal weight function.

Let $P(\boldsymbol{l}, n)$ be the set of sequences $I=\left(i_{1}^{1}, \ldots, i_{j_{1}}^{1} ; \ldots ; i_{1}^{n}, \ldots, i_{j_{n}}^{n}\right)$ of integers in $\{1, \ldots, r\}$ such that, for all $i=1, \ldots, r$, the integer $i$ appears in $I$ precisely $l_{i}$ times. For $I \in P(\boldsymbol{l}, n)$, and a permutation $\sigma \in \Sigma_{l}$, set $\sigma_{1}(i)=\sigma(i)$ for $i=1, \ldots, j_{1}$, and $\sigma_{s}(i)=\sigma\left(j_{1}+\cdots+j_{s-1}+i\right)$ for $s=2, \ldots, n$ and $i=1, \ldots, j_{s}$. Define

$$
\Sigma(I)=\left\{\sigma \in \Sigma_{l} \mid c\left(\sigma_{s}(j)\right)=i_{s}^{j} \text { for } s=1, \ldots, n \text { and } j=1, \ldots j_{s}\right\} .
$$

To every $I \in P(\boldsymbol{l}, n)$ we associate a vector

$$
F_{I} v=F_{i_{1}^{1}} \cdots F_{i_{j_{1}}^{1}} v_{\Lambda_{1}} \otimes \cdots \otimes F_{i_{1}^{n}} \cdots F_{i_{j_{n}}^{n}} v_{\Lambda_{n}}
$$

in $V_{\boldsymbol{\Lambda}}[\Lambda-\alpha(\boldsymbol{l})]$, and rational functions

$$
\omega_{I, \sigma}=\omega_{\sigma_{1}(1), \ldots, \sigma_{1}\left(j_{1}\right)}\left(z_{1}\right) \cdots \omega_{\sigma_{n}(1), \ldots, \sigma_{n}\left(j_{n}\right)}\left(z_{n}\right),
$$

labeled by $\sigma \in \Sigma(I)$, where

$$
\omega_{i_{1}, \ldots, i_{j}}\left(z_{s}\right)=\frac{1}{\left(t_{i_{1}}-t_{i_{2}}\right) \cdots\left(t_{i_{j-1}}-t_{i_{j}}\right)\left(t_{i_{j}}-z_{s}\right)} .
$$

We set

$$
\omega(z, t)=\sum_{I \in P(\boldsymbol{l}, n)} \sum_{\sigma \in \Sigma(I)} \omega_{I, \sigma} F_{I} v
$$




\section{E. Mukhin And A. Varchenko}

Examples. If $\boldsymbol{l}=(1,1,0, \ldots, 0)$, then

$$
\begin{aligned}
\omega(t, z)= & \frac{1}{\left(t_{1}-t_{2}\right)\left(t_{2}-z_{1}\right)} F_{1} F_{2} v_{\Lambda_{1}} \otimes v_{\Lambda_{2}}+\frac{1}{\left(t_{2}-t_{1}\right)\left(t_{1}-z_{1}\right)} F_{2} F_{1} v_{\Lambda_{1}} \otimes v_{\Lambda_{2}} \\
& +\frac{1}{\left(t_{1}-z_{1}\right)\left(t_{2}-z_{2}\right)} F_{1} v_{\Lambda_{1}} \otimes F_{2} v_{\Lambda_{2}}+\frac{1}{\left(t_{2}-z_{1}\right)\left(t_{1}-z_{2}\right)} F_{2} v_{\Lambda_{1}} \otimes F_{1} v_{\Lambda_{2}} \\
& +\frac{1}{\left(t_{1}-t_{2}\right)\left(t_{2}-z_{2}\right)} v_{\Lambda_{1}} \otimes F_{1} F_{2} v_{\Lambda_{2}}+\frac{1}{\left(t_{2}-t_{1}\right)\left(t_{1}-z_{2}\right)} v_{\Lambda_{1}} \otimes F_{2} F_{1} v_{\Lambda_{2}} .
\end{aligned}
$$

If $\boldsymbol{l}=(2,0, \ldots, 0)$, then

$$
\begin{aligned}
\omega(t, z)= & \left(\frac{1}{\left(t_{1}-t_{2}\right)\left(t_{2}-z_{1}\right)}+\frac{1}{\left(t_{2}-t_{1}\right)\left(t_{1}-z_{1}\right)}\right) F_{1}^{2} v_{\Lambda_{1}} \otimes v_{\Lambda_{2}} \\
& +\left(\frac{1}{\left(t_{1}-z_{1}\right)\left(t_{2}-z_{2}\right)}+\frac{1}{\left(t_{2}-z_{1}\right)\left(t_{1}-z_{2}\right)}\right) F_{1} v_{\Lambda_{1}} \otimes F_{1} v_{\Lambda_{2}} \\
& +\left(\frac{1}{\left(t_{1}-t_{2}\right)\left(t_{2}-z_{2}\right)}+\frac{1}{\left(t_{2}-t_{1}\right)\left(t_{1}-z_{2}\right)}\right) v_{\Lambda_{1}} \otimes F_{1}^{2} v_{\Lambda_{2}} .
\end{aligned}
$$

The universal weight function was introduced in [SV91] to solve the KZ equations; see [SV91, FSV95, FMTV00]. The hypergeometric solutions to the KZ equations with values in Sing $V_{\boldsymbol{\Lambda}}[\Lambda-\alpha(\boldsymbol{l})]$ have the form

$$
I(z)=\int_{\gamma(z)} \Phi(t, z, \boldsymbol{\Lambda}, \boldsymbol{l})^{1 / \kappa} \omega(t, z) d t .
$$

Lemma 2.1. Assume that $z \in \mathbb{C}^{n}$ has distinct coordinates. Assume that $t \in \mathbb{C}^{l}$ is a critical point of the master function $\Phi(., z, \boldsymbol{\Lambda}, \boldsymbol{l})$. Then the vector $\omega(t, z) \in V_{\boldsymbol{\Lambda}}[\Lambda-\alpha(\boldsymbol{l})]$ is well defined.

Proof. The rational function $\omega$ of $t$ and $z$ may have poles at hyperplanes given by equations of the form $t_{i}-t_{j}=0$ and $t_{i}-z_{s}=0$. All of the poles are of first order. We need to prove two facts:

(i) If $\left(\alpha_{c(i)}, \alpha_{c(j)}\right)=0$ for some $i$ and $j$, then $w$ does not have a pole at the hyperplane $t_{i}-t_{j}=0$.

(ii) If $\left(\alpha_{c(i)}, \Lambda_{s}\right)=0$ for some $i$ and $s$, then $w$ does not have a pole at the hyperplane $t_{i}-z_{s}=0$.

Assume that $\left(\alpha_{c(i)}, \alpha_{c(j)}\right)=0$ for some $i$ and $j$. From formulas for $\omega_{I, \sigma}$ it follows that the residue of $\omega$ at $t_{i}-t_{j}=0$ belongs to the span of the vectors in $V_{\boldsymbol{\Lambda}}$ having the form

$$
F_{i_{1}^{1}} \cdots F_{i_{j_{1}}^{1}} v_{\Lambda_{1}} \otimes \cdots \otimes F_{i_{1}^{s}} \cdots\left(F_{c(i)} F_{c(j)}-F_{c(j)} F_{c(i)}\right) \cdots F_{i_{j_{s}}^{s}} v_{\Lambda_{s}} \otimes \cdots \otimes F_{i_{1}^{n}} \cdots F_{i_{j_{n}}^{n}} v_{\Lambda_{n}} .
$$

But the element $F_{c(i)} F_{c(j)}-F_{c(j)} F_{c(i)}$ acts by zero on $V_{\boldsymbol{\Lambda}}$. Hence $\omega$ is regular at $t_{i}-t_{j}=0$.

Assume that $\left(\alpha_{c(i)}, \Lambda_{s}\right)=0$ for some $i$ and $s$. From formulas for $\omega_{I, \sigma}$ it follows that the residue of $\omega$ at $t_{i}-z_{s}=0$ belongs to the span of monomials

$$
F_{I} v=\cdots \otimes F_{i_{1}^{s}} \cdots F_{i_{j_{s}}^{s}} v_{\Lambda_{s}} \otimes \cdots
$$

such that $F_{i_{j_{s}}^{s}}=F_{c(i)}$. But $F_{c(i)} v_{\Lambda_{s}}=0$ in the irreducible $\mathfrak{g}$-module $V_{\Lambda_{s}}$. Hence $\omega$ is regular at $t_{i}-z_{s}=0$.

TheOrem 2.1. [RV95] Assume that $z \in \mathbb{C}^{n}$ has distinct coordinates. Assume that $t \in \mathbb{C}^{l}$ is a critical point of the master function $\Phi(., z, \boldsymbol{\Lambda}, \boldsymbol{l})$. Then the vector $\omega(t, z)$ belongs to Sing $V_{\boldsymbol{\Lambda}}[\Lambda-\alpha(\boldsymbol{l})]$ and is an eigenvector of the Gaudin hamiltonians $K_{1}(z), \ldots, K_{n}(z)$.

This theorem was proved in [RV95] using the quasi-classical asymptotics of the hypergeometric solutions of the KZ equations. The theorem also follows directly from Theorem 6.16.2 in [SV91]; cf. Theorem 7.2.5 in [SV91], and see also Theorem 4.2.2 in [FSV95].

The values of the universal weight function at the critical points (with respect to $t$ ) of the master function are called the Bethe vectors; see [RV95, Var95, FFR94]. 


\section{The Shapovalov form and iterated singular vectors}

\subsection{The Shapovalov form}

Define the anti-involution $\tau: \mathfrak{g} \rightarrow \mathfrak{g}$ sending $E_{1}, \ldots, E_{r}, H_{1}, \ldots, H_{r}$, and $F_{1}, \ldots, F_{r}$ to $F_{1}, \ldots, F_{r}$, $H_{1}, \ldots, H_{r}$, and $E_{1}, \ldots, E_{r}$, respectively.

Let $W$ be a highest weight $\mathfrak{g}$-module with highest weight vector $w$. The Shapovalov form on $W$ is the unique symmetric bilinear form $S$ defined by the conditions:

$$
S(w, w)=1, \quad S(x u, v)=S(u, \tau(x) v),
$$

for all $u, v \in W$ and $x \in \mathfrak{g}$; see [Kac90]. The Shapovalov form is non-degenerate on an irreducible $W$ and is positive definite on the real part of $W$.

Let $V_{\Lambda_{1}}, \ldots, V_{\Lambda_{n}}$ be irreducible highest weight modules and $V_{\boldsymbol{\Lambda}}$ their tensor product. Let $v_{\Lambda_{i}} \in$ $V_{\Lambda_{i}}$ be a highest weight vector and $S_{i}$ the corresponding Shapovalov form on $V_{\Lambda_{i}}$. Define a symmetric bilinear form on $V_{\boldsymbol{\Lambda}}$ by the formula

$$
S=S_{1} \otimes \cdots \otimes S_{n} .
$$

The form $S$ will be called the tensor Shapovalov form on $V_{\boldsymbol{\Lambda}}$.

Lemma 3.1 [RV95]. The Gaudin hamiltonians $K_{1}(z), \ldots, K_{n}(z)$ are symmetric with respect to $S$, $S\left(K_{i}(z) u, v\right)=S\left(u, K_{i}(z) v\right)$ for all $i, z, u, v$.

\subsection{Iterated singular vectors}

Let $n_{1}, \ldots, n_{k}$ be positive integers. For $p=0,1, \ldots, k$ fix a collection of non-negative integers $\boldsymbol{l}^{p}=\left(l_{1}^{p}, \ldots, l_{r}^{p}\right)$. Set $\boldsymbol{l}=\boldsymbol{l}^{0}+\boldsymbol{l}^{1}+\cdots+\boldsymbol{l}^{k}, \alpha\left(\boldsymbol{l}^{p}\right)=l_{1}^{p} \alpha_{1}+\cdots+l_{r}^{p} \alpha_{r}, n=n_{1}+\cdots+n_{k}, l^{p}=l_{1}^{p}+\cdots+l_{r}^{p}$, and $l=l^{0}+l^{1}+\cdots+l^{k}$. For $j=1, \ldots, r$, set $l_{j}=l_{j}^{0}+l_{j}^{1}+\cdots+l_{j}^{k}$. We have $l=l_{1}+\cdots+l_{r}$.

For $p=1, \ldots, k$ fix a collection of weights $\Lambda^{p}=\left(\Lambda_{1}^{p}, \Lambda_{2}^{p}, \ldots, \Lambda_{n_{p}}^{p}\right), \Lambda_{i}^{p} \in \mathfrak{h}^{*}$. Denote by $\boldsymbol{\Lambda}$ the collection of $n$ weights $\Lambda_{i}^{p}, p=1, \ldots, k, i=1, \ldots, n_{p}$. Set $\Lambda^{p}=\Lambda_{1}^{p}+\cdots+\Lambda_{n_{p}}^{p}, \Lambda=\Lambda^{1}+\cdots+\Lambda^{k}$. Set $\Lambda^{0}=\left(\Lambda_{1}^{0}, \ldots, \Lambda_{k}^{0}\right)$ where

$$
\Lambda_{p}^{0}=\Lambda^{p}-\alpha\left(\boldsymbol{l}^{p}\right)
$$

for $p=1, \ldots, k$. Set $\Lambda^{0}=\Lambda_{1}^{0}+\cdots+\Lambda_{k}^{0}$.

Consider the tensor products

$$
\begin{aligned}
V_{\Lambda^{0}} & =V_{\Lambda_{1}^{0}} \otimes \cdots \otimes V_{\Lambda_{k}^{0}}, \\
V_{\Lambda^{p}} & =V_{\Lambda_{1}^{p}} \otimes \cdots \otimes V_{\Lambda_{n_{p}}^{p}}, \quad \text { for } p=1, \ldots, k, \\
V_{\boldsymbol{\Lambda}} & =V_{\Lambda^{1}} \otimes \cdots \otimes V_{\Lambda^{k}} \\
& =V_{\Lambda_{1}^{1}} \otimes \cdots \otimes V_{\Lambda_{n_{1}}^{1}} \otimes \cdots \otimes V_{\Lambda_{1}^{k}} \otimes \cdots \otimes V_{\Lambda_{n_{k}}^{k}} .
\end{aligned}
$$

Let $S^{0}$ be the tensor Shapovalov form on $V_{\Lambda^{0}}, S^{p}$ the tensor Shapovalov form on $V_{\boldsymbol{\Lambda}^{p}}$, and $S=$ $S^{1} \otimes \cdots \otimes S^{k}$ the tensor Shapovalov form on $V_{\boldsymbol{\Lambda}}$.

To $p=1, \ldots, k$ and $I=\left(i_{1}^{1}, \ldots, i_{j_{1}}^{1} ; \ldots ; i_{1}^{n_{p}}, \ldots, i_{j_{n_{p}}}^{n_{p}}\right) \in P\left(\boldsymbol{l}^{p}, n_{p}\right)$ we associate a vector

$$
F_{I} v_{\Lambda^{p}}=F_{i_{1}^{1}} \cdots F_{i_{j_{1}}^{1}} v_{\Lambda_{1}^{p}} \otimes \cdots \otimes F_{i_{1}^{n_{p}}} \cdots F_{i_{j_{n}}^{n_{p}}} v_{\Lambda_{n_{p}}^{p}}
$$

in $V_{\Lambda^{p}}\left[\Lambda^{p}-\alpha\left(\boldsymbol{l}^{p}\right)\right]$. Assume that for $p=1, \ldots, k$ a singular vector

$$
w_{\boldsymbol{\Lambda}^{p}}=\sum_{I \in P\left(\boldsymbol{l}^{p}, n_{p}\right)} a_{I}^{p} F_{I} v_{\boldsymbol{\Lambda}^{p}} \in \operatorname{Sing} V_{\boldsymbol{\Lambda}^{p}}\left[\Lambda^{p}-\alpha\left(\boldsymbol{l}^{p}\right)\right]
$$

is chosen. Here $a_{I}^{p}$ are some complex numbers. 


\section{E. Mukhin And A. Varchenko}

To every $I=\left(i_{1}^{1}, \ldots, i_{j_{1}}^{1} ; \ldots ; i_{1}^{k}, \ldots, i_{j_{k}}^{k}\right) \in P\left(\boldsymbol{l}^{0}, k\right)$ we associate a vector

$$
F_{I} v_{\Lambda^{0}}=F_{i_{1}^{1}} \cdots F_{i_{j_{1}}^{1}} v_{\Lambda_{1}^{0}} \otimes \cdots \otimes F_{i_{1}^{k}} \cdots F_{i_{j_{k}}^{k}} v_{\Lambda_{k}^{0}}
$$

in $V_{\boldsymbol{\Lambda}^{0}}\left[\Lambda-\sum_{p=0}^{k} \alpha\left(\boldsymbol{l}^{p}\right)\right]$. Assume that a singular vector

$$
w_{\boldsymbol{\Lambda}^{0}}=\sum_{I \in P\left(\boldsymbol{l}^{0}, k\right)} a_{I}^{0} F_{I} v_{\boldsymbol{\Lambda}^{0}} \in \operatorname{Sing} V_{\boldsymbol{\Lambda}^{0}}\left[\Lambda-\sum_{p=0}^{k} \alpha\left(\boldsymbol{l}^{p}\right)\right]
$$

is chosen. Here $a_{I}^{0}$ are some complex numbers.

To every $I \in P\left(\boldsymbol{l}^{0}, k\right)$ we also associate a vector

$$
F_{I} w=F_{i_{1}^{1}} \cdots F_{i_{j_{1}}^{1}} w_{\boldsymbol{\Lambda}^{1}} \otimes \cdots \otimes F_{i_{1}^{k}} \cdots F_{i_{j_{k}}^{k}} w_{\boldsymbol{\Lambda}^{k}}
$$

in $V_{\boldsymbol{\Lambda}}\left[\Lambda-\sum_{p=0}^{k} \alpha\left(\boldsymbol{l}^{p}\right)\right]$. Here $F_{i_{1}^{p}} \cdots F_{i_{j_{p}}^{p}} w_{\boldsymbol{\Lambda}^{p}}$ denotes the action of $F_{i_{1}^{p}} \cdots F_{i_{j_{p}}^{p}}$ on the vector $w_{\boldsymbol{\Lambda}^{p}}$ in the $\mathfrak{g}$-module $V_{\Lambda^{p}}$.

The vector

$$
\boldsymbol{w}=\sum_{I \in P\left(\boldsymbol{l}^{0}, k\right)} a_{I}^{0} F_{I} w \in V_{\boldsymbol{\Lambda}}\left[\Lambda-\sum_{p=0}^{k} \alpha\left(\boldsymbol{l}^{p}\right)\right]
$$

is called the iterated singular vector with respect to the singular vectors $w_{\boldsymbol{\Lambda}^{0}}, w_{\boldsymbol{\Lambda}^{1}}, \ldots, w_{\boldsymbol{\Lambda}^{k}}$. It is easy to see that $\boldsymbol{w}$ is a singular vector in $V_{\boldsymbol{\Lambda}}$.

Lemma 3.2. We have

$$
S(\boldsymbol{w}, \boldsymbol{w})=\prod_{p=0}^{k} S^{p}\left(w_{\boldsymbol{\Lambda}^{p}}, w_{\boldsymbol{\Lambda}^{p}}\right)
$$

\section{Asymptotics of master functions and Bethe vectors}

\subsection{Asymptotics of master functions}

In this section we consider a master function $\Phi(t, z, \boldsymbol{\Lambda}, \boldsymbol{l})$ and assume that parameters $\boldsymbol{\Lambda}, \boldsymbol{l}$ do not change while $z$ depends on a complex parameter $\epsilon$. We assume that $z$ has a limit as $\epsilon$ tends to zero. We study the limit of the master function, its critical points, and its Bethe vectors as $\epsilon$ tends to zero.

We use the notation of $\S 3.2$.

Let $z=\left(z_{1}, \ldots, z_{n}\right)$. For $s=1, \ldots, n$ we assign the weight $\Lambda_{s-n_{1}-\cdots-n_{p-1}}^{p}$ to the coordinate $z_{s}$ if

$$
n_{1}+\cdots+n_{p-1}<s \leqslant n_{1}+\cdots+n_{p} .
$$

With this assignment we consider the master function $\Phi(t, z, \boldsymbol{\Lambda}, \boldsymbol{l})$ with $t=\left(t_{1}, \ldots, t_{l}\right)$.

Introduce the dependence of $z=\left(z_{1}, \ldots, z_{n}\right)$ on new variables $\epsilon$ and $\left(y_{i}^{p}\right)$ as follows. Let $y^{0}=$ $\left(y_{1}^{0}, \ldots, y_{k}^{0}\right)$. For $p=1, \ldots, k$, let $y^{p}=\left(y_{1}^{p}, \ldots, y_{n_{p}}^{p}\right)$. Let $y=\left(y_{i}^{p}\right)$ where $p=0, \ldots, k$ and $i=1, \ldots, k$ if $p=0$ and $i=1, \ldots, n_{p}$ if $p=1, \ldots, k$. Set

$$
z_{s}(y, \epsilon)=y_{p}^{0}+\epsilon y_{s-n_{1}-\cdots-n_{p-1}}^{p},
$$

if $s$ satisfies (7).

If the variables $y$ are fixed and $\epsilon \rightarrow 0$, then the coordinate $z_{s}(y, \epsilon)$ in (8) tends to $y_{p}^{0}$ and the ratio $\left(z_{s}(y, \epsilon)-y_{p}^{0}\right) / \epsilon$ has the limit $y_{s-n_{1}-\cdots-n_{p-1}}^{p}$.

Let $z=z(y, \epsilon)$ be the relation given by formula (8). 


\section{Norm of A Bethe vector AND the Hessian}

We rescale the variables $t$ of the master function $\Phi(t, z(y, \epsilon), \boldsymbol{\Lambda}, \boldsymbol{l})$ as follows. Introduce new variables $u=\left(u_{i}^{j}\right)$ where $j=0,1, \ldots, k$ and $i=1, \ldots, l^{j}$. If

$$
l_{1}+\cdots+l_{j-1}<i \leqslant l_{1}+\cdots+l_{j-1}+l_{j}^{0},
$$

then we set

$$
t_{i}=u_{l_{1}^{0}+\cdots+l_{j-1}^{0}+i-\left(l_{1}+\cdots+l_{j-1}\right)}^{0}
$$

If

then we set

$$
l_{1}+\cdots+l_{j-1}+l_{j}^{0}+\cdots+l_{j}^{p-1}<i \leqslant l_{1}+\cdots+l_{j-1}+l_{j}^{0}+\cdots+l_{j}^{p}
$$

$$
t_{i}=y_{p}^{0}+\epsilon u_{l_{1}^{p}+\cdots+l_{j-1}^{p}+i-\left(l_{1}+\cdots+l_{j-1}+l_{j}^{0}+\cdots+l_{j}^{p-1}\right)}^{p}
$$

Let $t=t(u, \epsilon)$ be the relation given by formulas (9) and (10). The relation $t=t(u, \epsilon)$, given by formulas (9) and (10), will be called the rescaling of variables $t$ with respect to the parameters $\boldsymbol{l}^{0}, \ldots, \boldsymbol{l}^{k}$ or simply the $\left(\boldsymbol{l}^{0}, \ldots, \boldsymbol{l}^{k}\right)$-type rescaling .

We study the asymptotics of the function $\Phi(t(u, \epsilon), z(y, \epsilon), \boldsymbol{\Lambda}, \boldsymbol{l})$ as $\epsilon$ tends to zero.

To describe the asymptotics we use the master functions $\Phi\left(u^{p}, y^{p}, \boldsymbol{\Lambda}^{p}, \boldsymbol{l}^{p}\right), p=0, \ldots, k$. Here $u^{p}=\left(u_{1}^{p}, \ldots, u_{l^{p}}^{p}\right)$ for $p=0, \ldots, k ; y^{0}=\left(y_{1}^{0}, \ldots, y_{k}^{0}\right) ; y^{p}=\left(y_{1}^{p}, \ldots, y_{n_{p}}^{p}\right)$ for $p=1, \ldots, k ; \boldsymbol{\Lambda}^{p}=$ $\left(\Lambda_{1}^{p}, \ldots, \Lambda_{n_{p}}^{p}\right)$ for $p=0, \ldots, k$; and $\boldsymbol{l}^{p}=\left(l_{1}^{p}, \ldots, l_{r}^{p}\right)$ for $p=0, \ldots, k$.

Lemma 4.1. Let all the parameters $\Lambda_{i}^{j}, l_{i}^{j}$ be fixed. Fix a compact subset $K \subset \mathbb{C}^{l} \times \mathbb{C}^{n}$ in the $(u, y)$-space such that the $y_{1}^{0}, \ldots, y_{k}^{0}$ coordinates of points in $K$ are distinct. Assume that $\epsilon$ tends to 0 . Then

$$
\Phi(t(u, \epsilon), z(y, \epsilon), \boldsymbol{\Lambda}, \boldsymbol{l})=\epsilon^{N\left(\boldsymbol{\Lambda}, \boldsymbol{l}^{1}, \ldots, \boldsymbol{l}^{k}\right)}(1+\mathcal{O}(\epsilon, u, y)) \prod_{p=0}^{k} \Phi\left(u^{p}, y^{p}, \boldsymbol{\Lambda}^{p}, \boldsymbol{l}^{p}\right) .
$$

Here $N\left(\boldsymbol{\Lambda}, \boldsymbol{l}^{1}, \ldots, \boldsymbol{l}^{k}\right)$ is a suitable constant. The function $\mathcal{O}(\epsilon, u, y)$ is holomorphic in $\mathbb{C} \times \mathbb{C}^{l} \times \mathbb{C}^{n}$ in a neighborhood of the set $\{0\} \times K$ and $\left.\mathcal{O}(\epsilon, u, y)\right|_{\epsilon=0}=0$.

\subsection{Asymptotics of critical points}

We keep the notation of $\S 4.1$.

Let $y^{0}(*)=\left(y_{1}^{0}(*), \ldots, y_{k}^{0}(*)\right)$ be a point in $\mathbb{C}^{k}$ with distinct coordinates. Let $u^{0}(*)=\left(u_{1}^{0}(*), \ldots\right.$, $\left.u_{l^{0}}^{0}(*)\right)$ be a non-degenerate critical point of the master function $\Phi\left(\cdot, y^{0}(*), \boldsymbol{\Lambda}^{0}, \boldsymbol{l}^{0}\right)$.

For $p=1, \ldots, k$ let $y^{p}(*)=\left(y_{1}^{p}(*), \ldots, y_{n_{p}}^{p}(*)\right)$ be a point in $\mathbb{C}^{n_{p}}$ with distinct coordinates. Let $u^{p}(*)=\left(u_{1}^{p}(*), \ldots, u_{l^{p}}^{p}(*)\right)$ be a non-degenerate critical point of the master function $\Phi\left(\cdot, y^{p}(*), \boldsymbol{\Lambda}^{p}, \boldsymbol{l}^{p}\right)$.

Lemma 4.2. There exist unique functions $u_{i}^{p}(\epsilon)$, where $p=0, \ldots, k$ and $i=1, \ldots, k$ if $p=0$ and $i=1, \ldots, n_{p}$ if $p=1, \ldots, k$, with the following properties.

(i) The functions $u_{i}^{p}(\epsilon)$ are holomorphic functions defined in a neighborhood of $\epsilon=0$ in $\mathbb{C}$.

(ii) We have $u_{i}^{p}(0)=u_{i}^{p}(*)$ for all $p, i$.

(iii) For all non-zero $\epsilon$ in a neighborhood of $\epsilon=0$ in $\mathbb{C}$ the point $u(\epsilon)=\left(u_{i}^{p}(\epsilon)\right)$ is a non-degenerate critical point of the function $\Phi(t(u, \epsilon), z(y(*), \epsilon), \boldsymbol{\Lambda}, \boldsymbol{l})$ with respect to the variables $u=\left(u_{i}^{p}\right)$.

Lemma 4.2 follows from Lemma 4.1 with the help of the implicit function theorem.

Let $u(\epsilon)$ be as in Lemma 4.2. Then for small non-zero $\epsilon$, the point $t(\epsilon)=t(u(\epsilon), \epsilon) \in \mathbb{C}^{l}$ is a non-degenerate critical point of the master function $\Phi(\cdot, z(y(*), \epsilon), \boldsymbol{\Lambda}, \boldsymbol{l})$. This family of critical 


\section{E. Mukhin And A. Varchenko}

points $t(\epsilon)$ of $\Phi(\cdot, z(y(*), \epsilon), \boldsymbol{\Lambda}, \boldsymbol{l})$ will be called the family of critical points associated with the $\left(\boldsymbol{l}^{0}, \ldots, \boldsymbol{l}^{k}\right)$-type rescaling and originated at the critical points $u^{0}(*), \ldots, u^{k}(*)$ of the master functions $\Phi\left(\cdot, y^{0}(*), \boldsymbol{\Lambda}^{0}, \boldsymbol{l}^{0}\right), \ldots, \Phi\left(\cdot, y^{k}(*), \boldsymbol{\Lambda}^{k}, \boldsymbol{l}^{k}\right)$, respectively.

\subsection{Asymptotics of Hessians}

If $f$ is a function of $t_{1}, \ldots, t_{n}$ and $t(*)=\left(t_{1}(*), \ldots, t_{n}(*)\right)$ is a point, then the determinant

$$
\operatorname{det}_{i, j=1, \ldots, n} \frac{\partial^{2} f}{\partial t_{i} \partial t_{j}}(t(*))
$$

is called the Hessian of $f$ at $t(*)$ with respect to variables $t=\left(t_{1}, \ldots, t_{n}\right)$ and is denoted by $\operatorname{Hess}_{t} f(t(*))$.

Lemma 4.3. Let $t(\epsilon)$ be the family of non-degenerate critical points of the master function $\Phi(\cdot, z(y(*), \epsilon), \boldsymbol{\Lambda}, \boldsymbol{l})$ associated with the $\left(\boldsymbol{l}^{0}, \ldots, \boldsymbol{l}^{k}\right)$-type rescaling and originated at the critical points $u^{0}(*), \ldots, u^{k}(*)$ of the master functions $\Phi\left(\cdot, y^{0}(*), \boldsymbol{\Lambda}^{0}, \boldsymbol{l}^{0}\right), \ldots, \Phi\left(\cdot, y^{k}(*), \boldsymbol{\Lambda}^{k}, \boldsymbol{l}^{k}\right)$, respectively. Then

$$
\lim _{\epsilon \rightarrow 0} \epsilon^{2\left(l^{1}+\cdots+l^{k}\right)} \operatorname{Hess}_{t} \log \Phi(t(\epsilon), z(y(*), \epsilon), \boldsymbol{\Lambda}, \boldsymbol{l})=\prod_{p=0}^{k} \operatorname{Hess}_{u^{p}} \log \Phi\left(u^{p}(*), y^{p}(*), \boldsymbol{\Lambda}^{p}, \boldsymbol{l}^{p}\right) .
$$

\subsection{Asymptotics of Bethe vectors}

Let $t(\epsilon)$ be the family of non-degenerate critical points of the master function $\Phi(\cdot, z(y(*), \epsilon), \boldsymbol{\Lambda}, \boldsymbol{l})$ associated with the $\left(\boldsymbol{l}^{0}, \ldots, \boldsymbol{l}^{k}\right)$-type rescaling and originated at the critical points $u^{0}(*), \ldots, u^{k}(*)$ of the master functions $\Phi\left(\cdot, y^{0}(*), \boldsymbol{\Lambda}^{0}, \boldsymbol{l}^{0}\right), \ldots, \Phi\left(\cdot, y^{k}(*), \boldsymbol{\Lambda}^{k}, \boldsymbol{l}^{k}\right)$, respectively.

Let

$$
\omega(t(\epsilon), z(y(*), \epsilon)) \in \operatorname{Sing} V_{\boldsymbol{\Lambda}}\left[\Lambda-\sum_{p=0}^{k} \alpha\left(\boldsymbol{l}^{p}\right)\right]
$$

be the Bethe vector corresponding to the critical point $t(\epsilon)$ of $\Phi(\cdot, z(y(*), \epsilon), \boldsymbol{\Lambda}, \boldsymbol{l})$.

For $p=0, \ldots, k$ let

$$
\omega\left(u^{p}(*), y^{p}(*)\right) \in V_{\Lambda^{p}}\left[\Lambda^{p}-\alpha\left(\boldsymbol{l}^{p}\right)\right]
$$

be the Bethe vector corresponding to the critical point $u^{p}(*)$ of $\Phi\left(\cdot, y^{p}(*), \boldsymbol{\Lambda}^{p}, \boldsymbol{l}^{p}\right)$.

Let

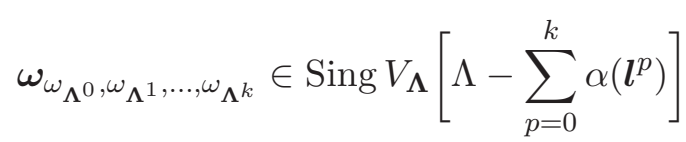

be the iterated singular vector with respect to singular vectors $\omega_{\boldsymbol{\Lambda}^{0}}, \omega_{\boldsymbol{\Lambda}^{1}}, \ldots, \omega_{\boldsymbol{\Lambda}^{k}}$.

LEMMA 4.4. We have

$$
\lim _{\epsilon \rightarrow 0} \epsilon^{l^{1}+\cdots+l^{k}} \omega(t(\epsilon), z(y(*), \epsilon))=\boldsymbol{\omega}_{\omega_{\boldsymbol{\Lambda}^{0}, \omega_{\Lambda^{1}}, \ldots, \omega_{\Lambda^{k}}} .}
$$

Lemma 4.4 easily follows from the formula for the universal weight function by repeated application of the identity

$$
\frac{1}{\left(t_{i}-t_{j}\right)\left(t_{j}-t_{k}\right)}=\frac{1}{\left(t_{i}-t_{k}\right)\left(t_{j}-t_{k}\right)}+\frac{1}{\left(t_{i}-t_{j}\right)\left(t_{i}-t_{k}\right)}
$$

\subsection{Asymptotics of hamiltonians}

In this section we keep the notation and assumptions of $\S 4.4$.

For $s=1, \ldots, n$, let $K_{s}(z): V_{\boldsymbol{\Lambda}} \rightarrow V_{\boldsymbol{\Lambda}}$ be the Gaudin hamiltonian associated with the tensor product $V_{\boldsymbol{\Lambda}}$ and the point $z \in \mathbb{C}^{n}$. Let $c_{s}(\epsilon)$ be the eigenvalue on the Bethe eigenvector $\omega(t(\epsilon), z(y(*), \epsilon))$ of the operator $K_{s}(z(y(*), \epsilon))$. 
For $i=1, \ldots, k$, let $K_{i}\left(y^{0}(*)\right): V_{\Lambda^{0}} \rightarrow V_{\Lambda^{0}}$ be the Gaudin hamiltonian associated with the tensor product $V_{\boldsymbol{\Lambda}^{0}}$ and the point $y^{0}(*) \in \mathbb{C}^{k}$. Let $c_{i}^{0}\left(u^{0}(*), y^{0}(*)\right)$ be the eigenvalue on the Bethe eigenvector $\omega\left(u^{0}(*), y^{0}(*)\right)$ of the operator $K_{i}\left(y^{0}(*)\right)$.

For $p=1, \ldots, k$ and $i=1, \ldots, n_{p}$, let $K_{i}\left(y^{p}(*)\right): V_{\Lambda^{p}} \rightarrow V_{\Lambda^{p}}$ be the Gaudin hamiltonian associated with the tensor product $V_{\boldsymbol{\Lambda}^{p}}$ and the point $y^{p}(*) \in \mathbb{C}^{n_{p}}$. Let $c_{i}^{p}\left(u^{p}(*), y^{p}(*)\right)$ be the eigenvalue on the Bethe eigenvector $\omega\left(u^{p}(*), y^{p}(*)\right)$ of the operator $K_{i}\left(y^{p}(*)\right)$.

Consider the tensor product $V_{\boldsymbol{\Lambda}}$ as the tensor product $V_{\boldsymbol{\Lambda}^{1}} \otimes \cdots \otimes V_{\boldsymbol{\Lambda}^{k}}$ of $k \mathfrak{g}$-modules. For $i=$ $1, \ldots, k$, consider the Gaudin hamiltonian $\widehat{K}_{i}\left(y^{0}(*)\right): V_{\boldsymbol{\Lambda}} \rightarrow V_{\boldsymbol{\Lambda}}$,

$$
\widehat{K}_{i}\left(y^{0}(*)\right)=\sum_{j=1, j \neq i}^{k} \frac{\Omega^{(i, j)}}{y_{i}^{0}(*)-y_{j}^{0}(*)},
$$

associated with those $k \mathfrak{g}$-modules and the point $y^{0}(*) \in \mathbb{C}^{k}$. For $p=1, \ldots, k$ and $i=1, \ldots, n_{p}$, denote by $\widehat{K}_{i}\left(y^{p}(*)\right)^{(p)}$ the linear operator on $V_{\boldsymbol{\Lambda}}=V_{\boldsymbol{\Lambda}^{1}} \otimes \cdots \otimes V_{\boldsymbol{\Lambda}^{k}}$ acting as $K_{i}\left(y^{p}(*)\right)$ on the factor $V_{\Lambda^{p}}$ and as the identity on other factors of that tensor product.

Lemma 4.5. Let $s \in\{1, \ldots, n\}$ and $s$ satisfies (7). If $n_{p}=1$, then

$$
\lim _{\epsilon \rightarrow 0} K_{s}\left(z\left(y^{0}(*), \epsilon\right)\right)=\widehat{K}_{p}\left(y^{0}(*)\right)
$$

and

If $n_{p}>1$, then

$$
\lim _{\epsilon \rightarrow 0} c_{i}(\epsilon)=c_{p}^{0}\left(u^{0}(*), y^{0}(*)\right)
$$

$$
\lim _{\epsilon \rightarrow 0} \epsilon K_{s}\left(z\left(y^{0}(*), \epsilon\right)\right)=\widehat{K}_{i-\left(n_{1}+\cdots+n_{p-1}\right)}\left(y^{p}(*)\right)^{(p)}
$$

and

$$
\lim _{\epsilon \rightarrow 0} \epsilon c_{i}(\epsilon)=c_{i-\left(n_{1}+\cdots+n_{p-1}\right)}^{p}\left(u^{p}(*), y^{p}(*)\right) .
$$

\section{Norms of Bethe vectors and Hessians}

\subsection{The $z$-dependence of the norm of a Bethe vector}

We use the notation of $\S 2.2$.

Fix a collection of weights $\boldsymbol{\Lambda}=\left(\Lambda_{1}, \ldots, \Lambda_{n}\right)$ and a collection of non-negative integers $\boldsymbol{l}=$ $\left(l_{1}, \ldots, l_{r}\right)$. Consider the master function $\Phi(t, z, \boldsymbol{\Lambda}, \boldsymbol{l})$.

Let $z^{0}=\left(z_{1}^{0}, \ldots, z_{n}^{0}\right)$ be a point with distinct coordinates. Let $t^{0}=\left(t_{1}^{0}, \ldots, t_{l}^{0}\right)$ be a nondegenerate critical point of the master function $\Phi\left(\cdot, z^{0}, \boldsymbol{\Lambda}, \boldsymbol{l}\right)$. By the implicit function theorem there exists a unique holomorphic $\mathbb{C}^{l}$-valued function $t=t(z)$, defined in the neighborhood of $z^{0}$ in $\mathbb{C}^{n}$, such that $t(z)$ is a non-degenerate critical point of the master function $\Phi(\cdot, z, \boldsymbol{\Lambda}, \boldsymbol{l})$ and $t\left(z^{0}\right)=t^{0}$. Let $\omega(t(z), z) \in \operatorname{Sing} V_{\boldsymbol{\Lambda}}[\Lambda-\alpha(\boldsymbol{l})]$ be the corresponding Bethe vector. Let $S$ be the tensor Shapovalov form on $V_{\boldsymbol{\Lambda}}$.

TheOREM 5.1 [RV95]. We have

$$
S(\omega(t(z), z), \omega(t(z), z))=C \operatorname{Hess}_{t} \log \Phi(t(z), z, \boldsymbol{\Lambda}, \boldsymbol{l}),
$$

where $C$ does not depend on $z$.

ConjeCture 5.1 [RV95]. The constant $C$ in (11) is equal to 1.

The conjecture is proved for $\mathfrak{g}=s l_{2}$ in [Var95]. We prove the conjecture for $\mathfrak{g}=s l_{r+1}$ in Theorem 7.1 . 


\section{E. Mukhin And A. Varchenko}

\subsection{The upper bound estimate for the number of critical points}

Fix a collection of integral dominant $\mathfrak{g}$-weights $\boldsymbol{\Lambda}=\left(\Lambda_{1}, \ldots, \Lambda_{n}\right)$ and a collection of non-negative integers $\boldsymbol{l}=\left(l_{1}, \ldots, l_{r}\right)$. Consider the master function $\Phi(t, z, \boldsymbol{\Lambda}, \boldsymbol{l})$ and its critical points with respect to $t$. Recall that the group $\Sigma_{\boldsymbol{l}}=\Sigma_{l_{1}} \times \cdots \times \Sigma_{l_{r}}$ acts on the critical set of $\Phi$.

TheOREM 5.2. If $\Lambda-\alpha(\boldsymbol{l})$ is not a dominant integral $\mathfrak{g}$-weight, then the master function $\Phi(\cdot, z, \boldsymbol{\Lambda}, \boldsymbol{l})$ does not have isolated critical points; see Corollary 5.3 in [MV03].

If $\Lambda-\alpha(\boldsymbol{l})$ is a dominant integral $\mathfrak{g}$-weight, then the master function $\Phi(\cdot, z, \boldsymbol{\Lambda}, \boldsymbol{l})$ has only isolated critical points; see Lemma 2.1 in [MV04].

If $\mathfrak{g}=s l_{r+1}$ and $\Lambda-\alpha(\boldsymbol{l})$ is a dominant integral sl $l_{r+1}$-weight, then the number of the $\boldsymbol{\Sigma}_{l^{-o r b i t s}}$ of critical points of the master function $\Phi(\cdot, z, \boldsymbol{\Lambda}, \boldsymbol{l})$, counted with multiplicities, is not greater than the multiplicity of the irreducible $s l_{r+1}$-module $V_{\Lambda-\alpha(l)}$ in the tensor product $V_{\boldsymbol{\Lambda}}$; see Theorem 5.13 in [MV04].

If $\mathfrak{g}=s l_{2}$, the weight $\Lambda-\alpha(\boldsymbol{l})$ is a dominant integral $s l_{2}$-weight, and the coordinates of the point $z=\left(z_{1}, \ldots, z_{n}\right)$ are generic, then the number of critical points of the master function $\Phi(\cdot, z, \boldsymbol{\Lambda}, \boldsymbol{l})$ is equal to the multiplicity of the irreducible $s l_{2}$-module $V_{\Lambda-\alpha(l)}$ in the tensor product $V_{\boldsymbol{\Lambda}}$. Moreover, in that case all critical points are non-degenerate; see Theorem 1 in [SV03].

\subsection{Tensor products of two $s l_{r+1}$-modules if one of them is fundamental}

Let $\lambda$ be an integral dominant $s l_{r+1}$-weight, $w_{1}, \ldots, w_{r}$ the fundamental $s l_{r+1}$-weights. Set $e_{1}=$ $w_{1}, e_{2}=w_{2}-w_{1}, \ldots, e_{r}=w_{r}-w_{r-1}, e_{r+1}=-w_{r}$. For $p=1, \ldots, r$ we have

$$
V_{\lambda} \otimes V_{w_{p}}=\bigoplus_{\mu} V_{\mu}
$$

where the sum is over all dominant integral weights $\mu$ such that $\mu=\lambda+e_{i_{1}}+\cdots+e_{i_{p}}, 1 \leqslant i_{1}<$ $\cdots<i_{p} \leqslant r+1$.

For example, if $\lambda, \mu$ are dominant integral $s l_{r+1}$-weights, then $V_{\mu}$ enters $V_{\lambda} \otimes V_{w_{1}}$ if and only if $\lambda=\mu-w_{1}+\sum_{j=1}^{i} \alpha_{j}$ for some $i \leqslant r$.

Notice also that if $\lambda, \mu$ are dominant integral $s l_{r+1}$-weights, then $V_{\mu}$ enters $V_{\lambda} \otimes V_{w_{r}}$ if and only if $\lambda=\mu-w_{r}+\sum_{j=i}^{r} \alpha_{j}$ for some $i \leqslant r$.

Consider the pair $\Lambda=\left(\Lambda_{1}, \Lambda_{2}\right)$ where $\Lambda_{1}$ is an integral dominant $s l_{r+1}$-weight, and $\Lambda_{2}=w_{1}$. Write $\Lambda_{1}=\sum_{j=1}^{r} \lambda_{j} w_{j}$ for suitable non-negative integers $\lambda_{j}$. Let $\boldsymbol{l}=\left(l_{1}, \ldots, l_{r}\right)=\left(1, \ldots, 1_{i}, 0_{i+1}\right.$, $\ldots, 0)$ for some $i \leqslant r$. Assume that $\mu=\Lambda_{1}+w_{1}-\alpha(\boldsymbol{l})$ is an integral dominant weight. Let $z^{0}=(0,1)$, and $t=\left(t_{1}, \ldots, t_{i}\right)$. Consider the master function $\Phi\left(t, z^{0}, \boldsymbol{\Lambda}, \boldsymbol{l}\right)$.

Let $S$ be the tensor Shapovalov form on $V_{\Lambda_{1}} \otimes V_{w_{1}}$.

Theorem 5.3 [MV00]. Under the above assumptions the function $\Phi\left(\cdot, z^{0}, \boldsymbol{\Lambda}, \boldsymbol{l}\right)$ has exactly one critical point, denoted by $t^{0}=\left(t_{1}^{0}, \ldots, t_{i}^{0}\right)$. The critical point $t^{0}$ is non-degenerate. The coordinates of $t^{0}$ are given by the formula

$$
t_{j}^{0}=\prod_{m=1}^{j} \frac{\lambda_{m}+\cdots+\lambda_{i}+i-m}{\lambda_{m}+\cdots+\lambda_{i}+i-m+1}, \quad j=1, \ldots, i .
$$

The Bethe vector $\omega\left(t^{0}, z^{0}\right) \in \operatorname{Sing} V_{\Lambda_{1}} \otimes V_{w_{1}}\left[\Lambda_{1}+w_{1}-\alpha(\boldsymbol{l})\right]$, corresponding to the critical point $t^{0}$, has the property

$$
S\left(\omega\left(t^{0}, z^{0}\right), \omega\left(t^{0}, z^{0}\right)\right)=\operatorname{Hess}_{t} \log \Phi\left(t^{0}, z^{0}, \boldsymbol{\Lambda}, \boldsymbol{l}\right) .
$$

Similarly consider the pair $\Lambda=\left(\Lambda_{1}, \Lambda_{2}\right)$ where $\Lambda_{1}$ is an integral dominant $s l_{r+1}$-weight, and $\Lambda_{2}=w_{r}$. Let $\boldsymbol{l}=\left(l_{1}, \ldots, l_{r}\right)=\left(0, \ldots, 0_{i}, 1_{i+1}, \ldots, 1\right)$ for some $i<r$. Assume that $\mu=\Lambda_{1}+w_{r}-\alpha(\boldsymbol{l})$ 


\section{Norm of A Bethe vector AND the Hessian}

is an integral dominant weight. Let $z^{0}=(0,1)$, and $t=\left(t_{1}, \ldots, t_{r-i}\right)$. Consider the master function $\Phi\left(t, z^{0}, \boldsymbol{\Lambda}, \boldsymbol{l}\right)$.

Let $S$ be the tensor Shapovalov form on the tensor product $V_{\Lambda_{1}} \otimes V_{w_{r}}$.

TheOREM 5.4 [MV00]. Under the above assumptions the function $\Phi\left(\cdot, z^{0}, \boldsymbol{\Lambda}, \boldsymbol{l}\right)$ has exactly one critical point, denoted by $t^{0}$. The critical point $t^{0}$ is non-degenerate. The Bethe vector $\omega\left(t^{0}, z^{0}\right)$ $\in$ Sing $V_{\Lambda_{1}} \otimes V_{w_{r}}\left[\Lambda_{1}+w_{r}-\alpha(\boldsymbol{l})\right]$, corresponding to the critical point $t^{0}$, has the property

$$
S\left(\omega\left(t^{0}, z^{0}\right), \omega\left(t^{0}, z^{0}\right)\right)=\text { Hess }_{t} \log \Phi\left(t^{0}, z^{0}, \boldsymbol{\Lambda}, \boldsymbol{l}\right) .
$$

The formulas for coordinates of the critical point in Theorem 5.4 can be easily deduced from formula (13).

\subsection{Tensor products of two $s l_{4}$-modules if one of them is the second fundamental}

If $\lambda, \mu$ are dominant integral $s l_{4}$-weights, then $V_{\mu}$ enters $V_{\lambda} \otimes V_{w_{2}}$ if and only if $\lambda=\mu-w_{2}+\delta$ where $\delta=0$ or $\delta$ is one of the following five weights:

$$
\alpha_{2}, \quad \alpha_{1}+\alpha_{2}, \quad \alpha_{2}+\alpha_{3}, \quad \alpha_{1}+\alpha_{2}+\alpha_{3}, \quad \alpha_{1}+2 \alpha_{2}+\alpha_{3} .
$$

For each $\delta$ in (14), write $\delta=l_{1} \alpha_{1}+l_{2} \alpha_{2}+l_{3} \alpha_{3}$ for suitable non-negative integers $l_{i}$. Set $\boldsymbol{l}=\left(l_{1}, l_{2}, l_{3}\right)$, $l=l_{1}+l_{2}+l_{3}, \boldsymbol{\Lambda}=\left(\lambda, w_{2}\right), z^{0}=(0,1)$, and $t=\left(t_{1}, \ldots, t_{l}\right)$.

Consider the master function $\Phi\left(t, z^{0}, \boldsymbol{\Lambda}, \boldsymbol{l}\right)$.

Theorem 5.5. Let $\lambda, \mu$ be dominant integral sl $l_{4}$-weights, such that $\lambda=\mu-w_{2}+\delta$ and $\delta$ is one of the weights in (14). Then the function $\Phi\left(\cdot, z^{0}, \boldsymbol{\Lambda}, \boldsymbol{l}\right)$ has exactly one critical point $t^{0}$. The critical point $t^{0}$ is non-degenerate. The Bethe vector $\omega\left(t^{0}, z^{0}\right) \in \operatorname{Sing} V_{\lambda} \otimes V_{w_{2}}[\mu]$, corresponding to $t^{0}$, is a non-zero vector.

Proof. If $\delta$ is $\alpha_{2}, \alpha_{1}+\alpha_{2}$, or $\alpha_{2}+\alpha_{3}$, then the theorem follows from Theorems 5.3 and 5.4.

If $\delta$ is $\alpha_{1}+\alpha_{2}+\alpha_{3}$ or $\alpha_{1}+2 \alpha_{2}+\alpha_{3}$, then the theorem is proved by direct verification. Namely, let $\lambda=\lambda_{1} w_{1}+\lambda_{2} w_{2}+\lambda_{3} w_{3}$. If $\delta=\alpha_{1}+\alpha_{2}+\alpha_{3}$, then one can check that $t^{0}=\left(t_{1}^{0}, t_{2}^{0}, t_{3}^{0}\right)$, where

$$
\begin{gathered}
t_{1}^{0}=\frac{\lambda_{1}\left(\lambda_{1}+\lambda_{2}+\lambda_{3}+2\right)}{\left(\lambda_{1}+1\right)\left(\lambda_{1}+\lambda_{2}+\lambda_{3}+3\right)}, \quad t_{2}^{0}=\frac{\lambda_{1}+\lambda_{2}+\lambda_{3}+2}{\lambda_{1}+\lambda_{2}+\lambda_{3}+3}, \\
t_{3}^{0}=\frac{\lambda_{3}\left(\lambda_{1}+\lambda_{2}+\lambda_{3}+2\right)}{\left(\lambda_{3}+1\right)\left(\lambda_{1}+\lambda_{2}+\lambda_{3}+3\right)} .
\end{gathered}
$$

If $\delta=\alpha_{1}+2 \alpha_{2}+\alpha_{3}$, then one can check that $t^{0}=\left(t_{1}^{0}, t_{2}^{0}, t_{3}^{0}, t_{4}^{0}\right)$, where

$$
\begin{gathered}
t_{1}^{0}=\frac{\left(\lambda_{1}+\lambda_{2}+1\right)\left(\lambda_{1}+\lambda_{2}+\lambda_{3}+2\right)}{\left(\lambda_{1}+\lambda_{2}+2\right)\left(\lambda_{1}+\lambda_{2}+\lambda_{3}+3\right)}, \quad t_{4}^{0}=\frac{\left(\lambda_{2}+\lambda_{3}+1\right)\left(\lambda_{1}+\lambda_{2}+\lambda_{3}+2\right)}{\left(\lambda_{2}+\lambda_{3}+2\right)\left(\lambda_{1}+\lambda_{2}+\lambda_{3}+3\right)}, \\
t_{2}^{0}+t_{3}^{0}-2=-\frac{\left(\lambda_{1}+2 \lambda_{2}+\lambda_{3}+4\right)\left(\lambda_{1} \lambda_{3}+2 \lambda_{1} \lambda_{2}+2 \lambda_{2} \lambda_{3}+2\left(\lambda_{2}\right)^{2}+2 \lambda_{1}+6 \lambda_{2}+2 \lambda_{3}+4\right)}{\left(\lambda_{2}+1\right)\left(\lambda_{1}+\lambda_{2}+2\right)\left(\lambda_{2}+\lambda_{3}+2\right)\left(\lambda_{1}+\lambda_{2}+\lambda_{3}+3\right)}, \\
t_{2}^{0} t_{3}^{0}=\frac{\lambda_{2}\left(\lambda_{1}+\lambda_{2}+1\right)\left(\lambda_{2}+\lambda_{3}+1\right)\left(\lambda_{1}+\lambda_{2}+\lambda_{3}+2\right)}{\left(\lambda_{2}+1\right)\left(\lambda_{1}+\lambda_{2}+2\right)\left(\lambda_{2}+\lambda_{3}+2\right)\left(\lambda_{1}+\lambda_{2}+\lambda_{3}+3\right)} .
\end{gathered}
$$

One easily verifies the statements of the theorem using those formulas.

\section{Critical points of the $s l_{r+1}$ master functions with first and last fundamental weights}

Let $\boldsymbol{\Lambda}=\left(\Lambda_{1}, \ldots, \Lambda_{n}\right)$ be a collection of $s l_{r+1}$-weights, each of which is either the first or last fundamental, i.e. $\Lambda_{i} \in\left\{w_{1}, w_{r}\right\}$. Let $\boldsymbol{l}=\left(l_{1}, \ldots, l_{r}\right)$ be a sequence of non-negative integers such that $\Lambda-\alpha(\boldsymbol{l})$ is integral dominant; here $\Lambda=\Lambda_{1}+\cdots+\Lambda_{n}$ and $\alpha(\boldsymbol{l})=l_{1} \alpha_{1}+\cdots+l_{r} \alpha_{r}$. 


\section{E. Mukhin And A. Varchenko}

Consider the master function $\Phi(t, z, \boldsymbol{\Lambda}, \boldsymbol{l})$ where $t=\left(t_{1}, \ldots, t_{l}\right), l=l_{1}+\cdots+l_{r}$, and $z=$ $\left(z_{1}, \ldots, z_{n}\right)$. Recall that the group $\boldsymbol{\Sigma}_{\boldsymbol{l}}=\Sigma_{l_{1}} \times \cdots \times \Sigma_{l_{r}}$ acts on the critical set of $\Phi(\cdot, z, \boldsymbol{\Lambda}, \boldsymbol{l})$.

TheOREM 6.1. For generic $z$ the following statements hold:

(i) The number of $\boldsymbol{\Sigma}_{\boldsymbol{l}}$-orbits of critical points of $\Phi(\cdot, z, \boldsymbol{\Lambda}, \boldsymbol{l})$ is equal to the multiplicity of the $s l_{r+1}$-module $V_{\Lambda-\alpha(l)}$ in the tensor product $V_{\boldsymbol{\Lambda}}$.

(ii) All critical points of $\Phi(\cdot, z, \boldsymbol{\Lambda}, \boldsymbol{l})$ are non-degenerate.

(iii) For every critical point $t^{0}$, the corresponding Bethe vector $\omega\left(t^{0}, z\right)$ has the property

$$
S\left(\omega\left(t^{0}, z\right), \omega\left(t^{0}, z\right)\right)=\operatorname{Hess}_{t} \log \Phi\left(t^{0}, z, \boldsymbol{\Lambda}, \boldsymbol{l}\right) .
$$

(iv) The Bethe vectors, corresponding to orbits of critical points of $\Phi(\cdot, z, \boldsymbol{\Lambda}, \boldsymbol{l})$, form a basis in Sing $V_{\boldsymbol{\Lambda}}[\Lambda-\alpha(\boldsymbol{l})]$.

Proof. The proof is by induction on $n$. If $n=2$, then the theorem follows from Theorems 5.3 and 5.4 .

Assume that Theorem 6.1 is proved for all tensor products of $n-1$ representations, each of which is either the first or last fundamental. We prove Theorem 6.1 for the tensor product $V_{\boldsymbol{\Lambda}}$ of $n$ given representations $V_{\Lambda_{1}}, \ldots, V_{\Lambda_{n}}$, each of which is either the first or last fundamental, and the given sequence $\boldsymbol{l}=\left(l_{1}, \ldots, l_{r}\right)$. We will use the notation and results of $\S \S 3.2$ and 4 .

We may assume that $\Lambda_{n}=w_{1}$. We may obtain that result by either reordering $\Lambda_{1}, \ldots, \Lambda_{n}$ or using the automorphism of $s l_{r+1}$ which sends $E_{i}, H_{i}, F_{i}, \alpha_{i}$, and $w_{i}$ to $E_{r+1-i}, H_{r+1-i}, F_{r+1-i}$, $\alpha_{r+1-i}$, and $w_{r+1-i}$, respectively.

Introduce $n_{1}, \ldots, n_{k}$, and $\boldsymbol{\Lambda}^{1}, \ldots, \boldsymbol{\Lambda}^{k}$ (as in $\S 3.2$ ) using the following formulas. Set $k=2$, $n_{1}=n-1, n_{2}=1, \Lambda^{1}=\left(\Lambda_{1}, \Lambda_{2}, \ldots, \Lambda_{n-1}\right), \Lambda^{2}=\left(\Lambda_{n}\right), V_{\Lambda^{1}}=V_{\Lambda_{1}} \otimes \cdots \otimes V_{\Lambda_{n-1}}, V_{\Lambda^{2}}=V_{\Lambda_{n}}$, and $V_{\boldsymbol{\Lambda}}=V_{\Lambda^{1}} \otimes V_{\Lambda^{2}}=V_{\Lambda_{1}} \otimes \cdots \otimes V_{\Lambda_{n-1}} \otimes V_{\Lambda_{n}}$.

Consider the set $M^{\prime}$ of the $r+1$ integral weights $\Lambda-w_{1}-\alpha(\boldsymbol{l}), \Lambda-w_{1}-\alpha(\boldsymbol{l})+\alpha_{1}, \ldots$, $\Lambda-w_{1}-\alpha(\boldsymbol{l})+\alpha_{1}+\cdots+\alpha_{r}$. Denote by $M$ the subset of all $\mu \in M^{\prime}$ which are dominant.

Denote by $\operatorname{mult}\left(\mu ; \lambda_{1}, \ldots, \lambda_{m}\right)$ the multiplicity of $V_{\mu}$ in $V_{\lambda_{1}} \otimes \cdots \otimes V_{\lambda_{m}}$. We have

$$
\operatorname{mult}\left(\Lambda-\alpha(\boldsymbol{l}) ; \Lambda_{1}, \ldots, \Lambda_{n}\right)=\sum_{\mu \in M} \operatorname{mult}\left(\mu ; \Lambda_{1}, \ldots, \Lambda_{n-1}\right) .
$$

To prove parts (i) and (ii) of the theorem we will introduce a dependence of $z$ on $\epsilon$ so that $z_{1}, \ldots, z_{n-1}$ tend to 0 as $\epsilon \rightarrow 0$ and $z_{n}$ tends to 1 . Using the results of $\S 4$ we will construct non-intersecting sets of $\boldsymbol{\Sigma}_{\boldsymbol{l}}$-orbits of critical points of $\Phi$, depending on $\epsilon$, labeled by $\mu \in M$, and consisting of mult $\left(\mu ; \Lambda_{1}, \ldots, \Lambda_{n-1}\right)$ elements each. Together with Theorem 5.2 it will prove parts (i) and (ii).

More precisely, introduce the dependence of $z=\left(z_{1}, \ldots, z_{n}\right)$ on the new variables $\epsilon$ and $y=$ $\left(y_{i}^{p}\right)=\left(y_{1}^{0}, y_{2}^{0}, y_{1}^{1}, \ldots, y_{n-1}^{1}\right)$ as follows. Set

$$
\begin{aligned}
& z_{s}(y, \epsilon)=y_{1}^{0}+\epsilon y_{s}^{1}, \quad s=1, \ldots, n-1, \\
& z_{n}(y, \epsilon)=y_{2}^{0} .
\end{aligned}
$$

Let $z=z(y, \epsilon)$ be the relation given by formula (15). Set $y^{0}=\left(y_{1}^{0}, y_{2}^{0}\right)$ and $y^{1}=\left(y_{1}^{1}, \ldots, y_{n-1}^{1}\right)$.

Introduce $r+1$ types of rescaling of coordinates $t$; cf. $\S 4.1$.

Type 0 rescaling. Set $\boldsymbol{l}^{0}=(0, \ldots, 0)$, and $\boldsymbol{l}^{1}=\left(l_{1}, \ldots, l_{r}\right)$. Introduce new variables $u=$ $\left(u_{1}^{1}, \ldots, u_{l}^{1}\right)$

$$
t_{i}=y_{1}^{0}+\epsilon u_{i}^{1}, \quad i=1, \ldots, l .
$$

This relation $t=t(u, \epsilon)$ will be called the type 0 rescaling of variables $t$. Set $u^{0}=\emptyset, u^{1}=\left(u_{1}^{1}, \ldots, u_{l}^{1}\right)$. 


\section{Norm of A Bethe vector AND the Hessian}

Type $m$ rescaling, $m=1, \ldots, r$. Set $\boldsymbol{l}^{0}=\left(1, \ldots, 1_{m}, 0, \ldots, 0\right)$, and $\boldsymbol{l}^{1}=\left(l_{1}-1, \ldots, l_{m}-1\right.$, $\left.l_{m+1}, \ldots, l_{r}\right)$. Introduce new variables $u=\left(u_{1}^{0}, \ldots, u_{m}^{0}, u_{1}^{1}, \ldots, u_{l-m}^{1}\right)$,

$$
\begin{array}{ll}
t_{i}=u_{j}^{0}, & \text { if } i=l_{1}+\cdots+l_{j-1}+1 \text { for } j=1, \ldots, m, \\
t_{i}=y_{1}^{0}+\epsilon u_{i-j}^{1}, & \text { if } l_{1}+\cdots+l_{j-1}+1<i \leqslant l_{1}+\cdots+l_{j} \text { for } j=1, \ldots, m, \\
t_{i}=y_{1}^{0}+\epsilon u_{i-m}^{1}, & \text { if } l_{1}+\cdots+l_{m}<i .
\end{array}
$$

This relation $t=t(u, \epsilon)$ will be called the type $m$ rescaling of variables $t$. Set $u^{0}=\left(u_{1}^{0}, \ldots, u_{m}^{0}\right)$, $u^{1}=\left(u_{1}^{1}, \ldots, u_{l-m}^{1}\right)$.

We study the asymptotics of the function $\Phi(t(u, \epsilon), z(y, \epsilon), \boldsymbol{\Lambda}, \boldsymbol{l})$ as $\epsilon$ tends to zero for each of the $r+1$ rescalings.

To describe the asymptotics we use the master functions $\Phi\left(u^{p}, y^{p}, \boldsymbol{\Lambda}^{p}, \boldsymbol{l}^{p}\right), p=0,1$. Here the collections $\boldsymbol{\Lambda}^{1}=\left(\Lambda_{1}, \Lambda_{2}, \ldots, \Lambda_{n-1}\right), \boldsymbol{l}^{0}, \boldsymbol{l}^{1}$, and the variables $u^{p}$ and $y^{p}$ have already been defined for each of the $r+1$ rescalings. The collection $\Lambda^{0}$ is defined as follows. For the type 0 rescaling we set $\boldsymbol{\Lambda}^{0}=\left(\Lambda^{1}-\alpha\left(\boldsymbol{l}^{1}\right), \Lambda_{n}\right)$. For the type $m$ rescaling with $m=1, \ldots, r$, we set $\boldsymbol{\Lambda}^{0}=\left(\Lambda^{1}-\alpha\left(\boldsymbol{l}^{1}\right)+\right.$ $\left.\alpha_{1}+\cdots+\alpha_{m}, \Lambda_{n}\right)$.

The master functions corresponding to the type $m$ rescaling will be provided with the corresponding index: $\Phi_{m}\left(u^{p}, y^{p}, \boldsymbol{\Lambda}^{p}, \boldsymbol{l}^{p}\right), p=0,1$.

Let $y^{1}(*)=\left(y_{1}^{1}(*), \ldots, y_{n-1}^{1}(*)\right)$ be a point with distinct coordinates such that the following holds:

For $m=0,1, \ldots, r$, if $\Lambda-w_{1}-\alpha(\boldsymbol{l})+\alpha_{1}+\cdots+\alpha_{m}$ is dominant, then the master function $\Phi_{m}\left(u^{1}, y^{1}(*), \boldsymbol{\Lambda}^{1}, \boldsymbol{l}^{1}\right)$ has $\operatorname{mult}\left(\Lambda-w_{1}-\alpha(\boldsymbol{l})+\alpha_{1}+\cdots+\alpha_{m} ; \Lambda_{1}, \ldots, \Lambda_{n-1}\right)$ distinct orbits of non-degenerate critical points satisfying parts (iii) and (iv) of Theorem 6.1.

Such $y^{1}(*)$ exists according to the induction assumptions.

Consider the type $m$ rescaling with $m=1, \ldots, r$. Put $y^{0}(*)=(0,1)$. By Theorem 5.3 the function $\Phi_{m}\left(\cdot, y^{0}(*), \Lambda^{0}, l^{0}\right)$ has one critical point. Denote the critical point by $u^{0}(*)=\left(u_{1}^{0}(*), \ldots, u_{m}^{0}(*)\right)$.

Choose $\operatorname{mult}\left(\Lambda-w_{1}-\alpha(\boldsymbol{l})+\alpha_{1}+\cdots+\alpha_{m} ; \Lambda_{1}, \ldots, \Lambda_{n-1}\right)$ critical points of $\Phi_{p}\left(\cdot, y^{1}(*), \boldsymbol{\Lambda}^{1}, \boldsymbol{l}^{1}\right)$ lying in different $\Sigma_{l_{1}-1} \times \cdots \times \Sigma_{l_{m}-1} \times \Sigma_{l_{m+1}} \times \cdots \times \Sigma_{l_{r}}$-orbits. Denote those critical points by $u^{1}\left(*_{j}\right)$, $j=1, \ldots, \operatorname{mult}\left(\Lambda-w_{1}-\alpha(\boldsymbol{l})+\alpha_{1}+\cdots+\alpha_{m} ; \Lambda_{1}, \ldots, \Lambda_{n-1}\right)$. Let $t(\epsilon, j, m) \in \mathbb{C}^{l}$ be the family of critical points of $\Phi(\cdot, z(y(*), \epsilon), \boldsymbol{\Lambda}, \boldsymbol{l})$ associated with type $m$ rescaling and originated at the critical points $u^{0}(*)$, and $u^{1}\left(*_{j}\right)$ of the master functions $\Phi_{m}\left(\cdot, y^{0}(*), \boldsymbol{\Lambda}^{0}, \boldsymbol{l}^{0}\right)$ and $\Phi_{m}\left(\cdot, y^{1}(*), \boldsymbol{\Lambda}^{1}, \boldsymbol{l}^{1}\right)$, respectively; see $\S 4.2$.

Consider the type 0 rescaling. Put $y^{0}(*)=(0,1)$. The function $\Phi_{0}\left(u^{0}, y^{0}(*), \boldsymbol{\Lambda}^{0}, \boldsymbol{l}^{0}\right)$ does not depend on $u^{0}$.

Choose $\operatorname{mult}\left(\Lambda-w_{1}-\alpha(\boldsymbol{l}) ; \Lambda_{1}, \ldots, \Lambda_{n-1}\right)$ critical points of $\Phi_{0}\left(\cdot, y^{1}(*), \boldsymbol{\Lambda}^{1}, \boldsymbol{l}^{1}\right)$ lying in different $\Sigma_{l_{1}} \times \cdots \times \Sigma_{l_{r}}$-orbits. Denote the critical points by $u^{1}\left(*_{j}\right), j=1, \ldots, \operatorname{mult}\left(\Lambda-w_{1}-\right.$ $\left.\alpha(\boldsymbol{l}) ; \Lambda_{1}, \ldots, \Lambda_{n-1}\right)$. Let $t(\epsilon, j, 0) \in \mathbb{C}^{l}$ be the family of critical points of $\Phi(\cdot, z(y(*), \epsilon), \boldsymbol{\Lambda}, \boldsymbol{l})$ associated with type 0 rescaling and originated at the critical point $u^{1}\left(*_{j}\right)$ of the master function $\Phi_{0}\left(\cdot, y^{1}(*), \boldsymbol{\Lambda}^{1}, \boldsymbol{l}^{1}\right)$; see $\S 4.2$.

All together we have constructed $\operatorname{mult}\left(\Lambda-\alpha(\boldsymbol{l}) ; \Lambda_{1}, \ldots, \Lambda_{n}\right)$ families of critical points of $\Phi(\cdot, z(y(*), \epsilon), \boldsymbol{\Lambda}, \boldsymbol{l})$.

The constructed families are all different. Indeed, the families corresponding to the same rescaling are different by construction. The families corresponding to different rescalings are different because they have different limits as $\epsilon$ tends to 0 . Now Theorem 5.2 implies part (i).

All constructed critical points are non-degenerate by Lemma 4.2. This proves part (ii). Part (iii) is a direct corollary of the induction assumptions, Theorems 5.1 and 5.3, and Lemmas 4.4 and 4.3.

Part (iv) is a direct corollary of the construction and Lemma 4.4 . 


\section{E. Mukhin And A. Varchenko}

Let $\boldsymbol{\Lambda}=\left(\Lambda_{1}, \ldots, \Lambda_{n}\right)$ be a collection of $s l_{4}$-weights, each of which is fundamental, i.e. $\Lambda_{i} \in$ $\left\{w_{1}, w_{2}, w_{3}\right\}$. Let $\boldsymbol{l}=\left(l_{1}, l_{2}, l_{3}\right)$ be a sequence of non-negative integers such that $\Lambda-\alpha(\boldsymbol{l})$ is integral dominant; here $\Lambda=\Lambda_{1}+\cdots+\Lambda_{n}$ and $\alpha(\boldsymbol{l})=l_{1} \alpha_{1}+l_{2} \alpha_{2}+l_{3} \alpha_{3}$.

Consider the master function $\Phi(t, z, \boldsymbol{\Lambda}, \boldsymbol{l})$ where $t=\left(t_{1}, \ldots, t_{l}\right), l=l_{1}+l_{2}+l_{3}$, and $z=$ $\left(z_{1}, \ldots, z_{n}\right)$. Recall that the group $\boldsymbol{\Sigma}_{\boldsymbol{l}}=\Sigma_{l_{1}} \times \Sigma_{l_{2}} \times \Sigma_{l_{3}}$ acts on the critical set of $\Phi(\cdot, z, \boldsymbol{\Lambda}, \boldsymbol{l})$.

TheOREM 6.2. For generic $z$ the following statements hold:

(i) the number of $\boldsymbol{\Sigma}_{\boldsymbol{l}}$-orbits of critical points of $\Phi(\cdot, z, \boldsymbol{\Lambda}, \boldsymbol{l})$ is equal to the multiplicity of the $s l_{4}$-module $V_{\Lambda-\alpha(l)}$ in the tensor product $V_{\boldsymbol{\Lambda}}$;

(ii) all critical points of $\Phi(\cdot, z, \boldsymbol{\Lambda}, \boldsymbol{l})$ are non-degenerate;

(iii) the Bethe vectors, corresponding to orbits of critical points of $\Phi(\cdot, z, \boldsymbol{\Lambda}, \boldsymbol{l})$, are non-zero vectors and form a basis in Sing $V_{\boldsymbol{\Lambda}}[\Lambda-\alpha(\boldsymbol{l})]$.

The proof of this theorem is parallel to the proof of Theorem 6.1 and is based on Theorem 5.5.

\section{Norms of Bethe vectors in the $s l_{r+1}$ Gaudin models}

Let $\Lambda^{0}=\left(\Lambda_{1}^{0}, \ldots, \Lambda_{k}^{0}\right)$ be a collection of $s l_{r+1}$ integral dominant weights. Let $\boldsymbol{l}^{0}=\left(l_{1}^{0}, \ldots, l_{r}^{0}\right)$ be a sequence of non-negative integers such that $\Lambda^{0}-\alpha\left(\boldsymbol{l}^{0}\right)$ is integral dominant. Here $\Lambda^{0}=\Lambda_{1}^{0}+\cdots+\Lambda_{n}^{0}$ and $\alpha\left(\boldsymbol{l}^{0}\right)=l_{1}^{0} \alpha_{1}+\cdots+l_{r}^{0} \alpha_{r}$.

Consider the master function $\Phi\left(u^{0}, y^{0}, \boldsymbol{\Lambda}^{0}, \boldsymbol{l}^{0}\right)$ where $u^{0}=\left(u_{1}^{0}, \ldots, u_{l^{0}}^{0}\right), l^{0}=l_{1}^{0}+\cdots+l_{r}^{0}$, and $y^{0}=\left(y_{1}^{0}, \ldots, y_{k}^{0}\right)$.

Theorem 7.1. Let $y^{0}(*) \in \mathbb{C}^{k}$ be a point with distinct coordinates. Let $u^{0}(*)$ be a non-degenerate critical point of $\Phi\left(\cdot, y^{0}(*), \boldsymbol{\Lambda}^{0}, \boldsymbol{l}^{0}\right)$. Let $\omega\left(u^{0}(*), y^{0}(*)\right) \in \operatorname{Sing} V_{\boldsymbol{\Lambda}^{0}}\left[\Lambda^{0}-\alpha\left(\boldsymbol{l}^{0}\right)\right]$ be the corresponding Bethe vector. Let $S^{0}$ be the tensor Shapovalov form on $V_{\Lambda^{0}}$. Then

$$
S^{0}\left(\omega\left(u^{0}(*), y^{0}(*)\right), \omega\left(u^{0}(*), y^{0}(*)\right)\right)=\operatorname{Hess}_{u^{0}} \log \Phi\left(u^{0}(*), y^{0}(*), \boldsymbol{\Lambda}^{0}, \boldsymbol{l}^{0}\right) .
$$

Corollary 7.1. The Bethe vector $\omega\left(u^{0}(*), y^{0}(*)\right)$ is a non-zero vector.

Proof of Theorem 7.1. We deduce Theorem 7.1 from Theorem 6.1 using the results of $\S 4$.

It is known that for each integral dominant $s l_{r+1}$-weight $\lambda$, the multiplicity of $V_{\lambda}$ in $V_{w_{1}}^{\otimes n}$ is positive for a suitable $n$.

For each $p=1, \ldots, k$, fix $n_{p}$ such that the multiplicity of $V_{\Lambda_{p}^{0}}$ in $V_{w_{1}}^{\otimes n_{p}}$ is positive. Set $\Lambda^{p}=$ $\left(w_{1}, \ldots, w_{1}\right)$ where $w_{1}$ is taken $n_{p}$ times. Denote by $S^{p}$ the tensor product Shapovalov form on $V_{w_{1}}^{\otimes n_{p}}$.

We have $n_{p} w_{1}-\Lambda_{p}^{0}=l_{1}^{p} \alpha_{1}+\cdots+l_{r}^{p} \alpha_{r}$ where $\boldsymbol{l}^{p}=\left(l_{1}^{p}, \ldots, l_{r}^{p}\right)$ is a sequence of non-zero integers. Set $l^{p}=l_{1}^{p}+\cdots+l_{r}^{p}, y^{p}=\left(y_{1}^{p}, \ldots, y_{n_{p}}^{p}\right)$, and $u^{p}=\left(u_{1}^{p}, \ldots, u_{l p}^{p}\right)$. Consider the master function $\Phi\left(u^{p}, y^{p}, \boldsymbol{\Lambda}^{p}, \boldsymbol{l}^{p}\right)$. That master function satisfies the conditions of Theorem 6.1. Hence there exists a point $y^{p}(*) \in \mathbb{C}^{n_{p}}$ with distinct coordinates and a non-degenerate critical point $u^{p}(*) \in \mathbb{C}^{l^{p}}$ of the function $\Phi\left(\cdot, y^{p}(*), \Lambda^{p}, \boldsymbol{l}^{p}\right)$ such that the Bethe vector $\omega\left(u^{p}(*), y^{p}(*)\right) \in \operatorname{Sing} V_{w_{1}}^{\otimes n_{p}}\left[\Lambda_{p}^{0}\right]$ satisfies the identity:

$$
S^{p}\left(\omega\left(u^{p}(*), y^{p}(*)\right), \omega\left(u^{p}(*), y^{p}(*)\right)\right)=\operatorname{Hess}_{u^{p}} \log \Phi\left(u^{p}(*), y^{p}(*), \boldsymbol{\Lambda}^{p}, \boldsymbol{l}^{p}\right) .
$$

Set $n=n_{1}+\cdots+n_{k}, \boldsymbol{l}=\boldsymbol{l}^{0}+\cdots+\boldsymbol{l}^{k}=\left(l_{1}^{0}+\cdots+l_{1}^{k}, \ldots, l_{r}^{0}+\cdots+l_{r}^{k}\right), l=l^{0}+\cdots+l^{k}$. Set $z=\left(z_{i}^{p}\right)$, where $p=1, \ldots, k, i=1, \ldots, n_{p}$. Set $\boldsymbol{\Lambda}=\left(\Lambda_{i}^{p}\right)$, where $p=1, \ldots, k, i=1, \ldots, n_{p}$, and $\Lambda_{i}^{p}=w_{1}$. Assign the weight $\Lambda_{i}^{p}$ to the variable $z_{i}^{p}$ for every $p$, $i$. Set $t=\left(t_{1}, \ldots, t_{l}\right)$. Consider the master function $\Phi(t, z, \boldsymbol{\Lambda}, \boldsymbol{l})$. 


\section{Norm of A Bethe vector And the Hessian}

Introduce the dependence of variables $z$ on variables $u, \epsilon$ by the formula: $z_{i}^{p}=y_{p}^{0}+\epsilon y_{i}^{p}$ for all $p, i$. Introduce the $\left(\boldsymbol{l}^{0}, \ldots, \boldsymbol{l}^{k}\right)$ rescaling of variables $t$ by formulas $(9)$ and $(10)$. Let $t(\epsilon) \in \mathbb{C}^{l}$ be the family of critical points associated with this rescaling and originated at the critical points $u^{0}(*), \ldots, u^{k}(*)$ of the master functions $\Phi\left(\cdot, y^{0}(*), \boldsymbol{\Lambda}^{0}, \boldsymbol{l}^{0}\right), \ldots, \Phi\left(\cdot, y^{k}(*), \boldsymbol{\Lambda}^{k}, \boldsymbol{l}^{k}\right)$, respectively; see $\S 4.2$.

Let $\omega(t(\epsilon), z(y(*), \epsilon)) \in \operatorname{Sing} V_{w_{1}}^{\otimes n}$ be the corresponding Bethe vector. Let $S$ be the tensor Shapovalov form on $V_{w_{1}}^{\otimes n}$. By Theorem 6.1 we have

$$
S(\omega(t(\epsilon), z(y(*), \epsilon)), \omega(t(\epsilon), z(y(*), \epsilon)))=\operatorname{Hess}_{t} \log \Phi(\omega(t(\epsilon), z(y(*), \epsilon)), \boldsymbol{\Lambda}, \boldsymbol{l}) .
$$

Now by Lemmas $4.3,4.4$, and 3.2 we may conclude that

$$
S^{0}\left(\omega\left(u^{0}(*), y^{0}(*)\right), \omega\left(u^{0}(*), y^{0}(*)\right)\right)=\operatorname{Hess}_{u^{0}} \log \Phi\left(u^{0}(*), y^{0}(*), \boldsymbol{\Lambda}^{0}, \boldsymbol{l}^{0}\right) .
$$

Similarly to Theorem 7.1 one can prove the following theorem.

Theorem 7.2. Let $t^{0}(*)$ be a critical point of $\Phi\left(\cdot, y^{0}(*), \boldsymbol{\Lambda}^{0}, \boldsymbol{l}^{0}\right)$. Let $\omega\left(u^{0}(*), y^{0}(*)\right) \in \operatorname{Sing} V_{\boldsymbol{\Lambda}^{0}}\left[\Lambda^{0}-\right.$ $\left.\alpha\left(\boldsymbol{l}^{0}\right)\right]$ be the corresponding Bethe vector. Assume that the number

$$
S^{0}\left(\omega\left(u^{0}(*), y^{0}(*)\right), \omega\left(u^{0}(*), y^{0}(*)\right)\right)
$$

is not equal to zero. Then $t^{0}(*)$ is a non-degenerate critical point.

Corollary 7.2. Let $t^{0}(*)$ be a critical point of $\Phi\left(\cdot, y^{0}(*), \boldsymbol{\Lambda}^{0}, \boldsymbol{l}^{0}\right)$ such that the corresponding Bethe vector $\omega\left(u^{0}(*), y^{0}(*)\right) \in \operatorname{Sing} V_{\Lambda^{0}}\left[\Lambda^{0}-\alpha\left(\boldsymbol{l}^{0}\right)\right]$ is not equal to zero and belongs to the real part of $V_{\Lambda^{0}}$. Then $t^{0}(*)$ is a non-degenerate critical point.

The corollary follows from Theorem 7.2 since the Shapovalov form is positive definite on the real part of $V_{\Lambda^{0}}$.

Example (cf. [RV95]). Let $\mathfrak{g}=s l_{2}, \boldsymbol{\Lambda}^{0}=\left(w_{1}, w_{1}, w_{1}\right), \boldsymbol{l}^{0}=(1)$, and $y^{0}(*)=\left(1, \eta, \eta^{2}\right)$, where $\eta=e^{2 \pi i / 3}$. Consider the master function $\Phi\left(t, y^{0}(*), \boldsymbol{\Lambda}^{0}, \boldsymbol{l}^{0}\right)=\left(\left(t_{1}\right)^{3}-1\right)^{-1}$. The point $t^{0}(*)=(0)$ is the only critical point of $\Phi$. The critical point is degenerate. The corresponding Bethe vector

$$
\begin{aligned}
\omega\left(u^{0}(*), y^{0}(*)\right)= & -F_{1} v_{w_{1}} \otimes v_{w_{1}} \otimes v_{w_{1}} \\
& -\eta^{2} v_{w_{1}} \otimes F_{1} v_{w_{1}} \otimes v_{w_{1}}-\eta v_{w_{1}} \otimes v_{w_{1}} \otimes F_{1} v_{w_{1}} \in V_{\Lambda^{0}}
\end{aligned}
$$

is a non-zero vector and $S^{0}\left(\omega\left(u^{0}(*), y^{0}(*)\right), \omega\left(u^{0}(*), y^{0}(*)\right)\right)=1+\eta^{4}+\eta^{2}=0$.

\section{REFERENCES}

Bab93 H. Babujian, Off-shell Bethe ansatz equations and $N$-point correlators in the $\mathrm{SU}(2)$ WZNW theory, J. Phys. A 26 (1993), 6981-6990.

BF94 H. Babujian and R. Flume, Off-shell Bethe ansatz equation for Gaudin magnets and solutions of Knizhnik-Zamolodchikov equations, Modern Phys. Lett. A 9 (1994), 2029-2039.

BIK93 N. M. Bogoliubov, A. G. Izergin and V. E. Korepin, Quantum inverse scattering method and correlation functions (Cambridge University Press, Cambridge, 1993).

EH83 D. Eisenbud and J. Harris, Divisors on general curves and cuspidal rational curves, Invent. Math. 74 (1983), 371-418.

Fad90 L. D. Faddeev, Lectures on quantum inverse scattering method, in Integrable systems, Nankai Lectures Math. Phys., ed. X.-C. Song (World Scientific, Singapore, 1990), 23-70.

FFR94 B. Feigin, E. Frenkel and N. Reshetikhin, Gaudin model, Bethe ansatz and critical level, Comm. Math. Phys. 166 (1994), 27-62. 


\section{Norm of A Bethe vector And the Hessian}

FMTV00 G. Felder, Y. Markov, V. Tarasov and A. Varchenko, Differential equations compatible with KZ equations, Math. Phys. Anal. Geom. 3 (2000), 139-177.

Fre95 E. Frenkel, Affine algebras, Langlands duality and Bethe ansatz, in XIth International Congress of Mathematical Physics (Paris, 1994) (Internat. Press, Cambridge, MA, 1995), 606-642.

Fre04 E. Frenkel, Opers on the projective line, flag manifolds and Bethe ansatz, Mosc. Math. J. 4 (2004), 655-705, 783.

FSV95 B. Feigin, V. Schechtman and A. Varchenko, On algebraic equations satisfied by hypergeometric correlators in WZW models. II, Comm. Math. Phys. 170 (1995), 219-247.

FT79 L. D. Faddeev and L. A. Takhtajan, Quantum inverse problem method and the Heisenberg XYZ-model, Russian Math. Surveys 34 (1979), 11-68.

Gau76 M. Gaudin, Diagonalisation d'une classe d'Hamiltoniens de spin, J. Physique 37 (1976), 1089-1098.

Kac90 V. Kac, Infinite-dimensional Lie algebras (Cambridge University Press, Cambridge, 1990).

Kor82 V. Korepin, Calculation of norms of Bethe wave functions, Comm. Math. Phys. 86 (1982), 391-418.

MV00 E. Mukhin and A. Varchenko, Remarks on critical points of phase functions and norms of Bethe vectors, Adv. Stud. Pure Math. 27 (2000), 239-246.

MV03 E. Mukhin and A. Varchenko, Miura opers and critical points of master functions, Preprint (2003), math.QA/0312406.

MV04 E. Mukhin and A. Varchenko, Critical points of master functions and flag varieties, Commun. Contemp. Math. 6 (2004), 111-163.

Res86 N. Reshetikhin, Calculation of the norm of Bethe vectors in models with SU(3) symmetry, Zap. Nauchn. Sem. Leningrad. Otdel. Mat. Inst. Steklov. (LOMI) 150 (1986), 196-213.

RV95 N. Reshetikhin and A. Varchenko, Quasiclassical asymptotics of solutions to the KZ equations, in Geometry, topology and physics for R. Bott (Internat. Press, Cambridge, MA, 1995), 293-322.

Sot99 F. Sottile, The special Schubert calculus is real, Electron. Res. Announc. Amer. Math. Soc. 5 (1999), 35-39.

SV91 V. Schechtman and A. Varchenko, Arrangements of hyperplanes and Lie algebra homology, Invent. Math. 106 (1991), 139-194.

SV03 I. Scherbak and A. Varchenko, Critical points of functions, $\mathfrak{s l}_{2}$ representations, and Fuchsian differential equations with only univalued solutions, Mosc. Math. J. 3 (2003), 621-645.

TV96 V. Tarasov and A. Varchenko, Asymptotic solutions to the quantized Knizhnik-Zamolodchikov equation and Bethe vectors, Amer. Math. Soc. Transl. Ser. 2174 (1996), 235-273.

Var95 A. Varchenko, Critical points of the product of powers of linear functions and families of bases of singular vectors, Compositio Math. 97 (1995), 385-401.

Evgeny Mukhin mukhin@math.iupui.edu

Department of Mathematical Sciences, Indiana University-Purdue University Indianapolis, 402 North Blackford St., Indianapolis, IN 46202-3216, USA

Alexander Varchenko anv@email.unc.edu

Department of Mathematics, University of North Carolina at Chapel Hill, Chapel Hill, NC 275993250 , USA 